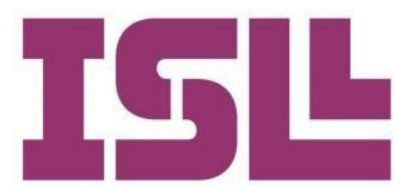

Número 11

Junio de 2019

\title{
La proxemia en la lectura: cómo se aproximan al texto los lectores de primaria según su edad, contexto y nivel de competencia lectora
}

\author{
Sara Recio-Pineda \\ Universidad de Barcelona \\ https://orcid.org/0000-0002-7823-3504
}

Recibido: 11-01-2019

Aceptado: 25-06-2019

Pág. 1 a la 18

\section{Palabras clave:}

proxemia, lectura, educación primaria, código postural, competencia lectora

\section{Resumen:}

Algunos lectores se alejan del texto que proxémicas con distintas versiones. Se deben leer; otros se asoman a él desde descubrió que la mayoría de lectores de la sus sillas; otros se acercan, lo tapan, lo muestra optaban por asomarse al texto, tocan, lo rodean, y otros muchos se aunque los lectores que, de acuerdo a sus apropian de él, lo cogen o se lo acercan. maestros, presentaban un nivel de En los últimos años, el estudio de la competencia lectora alta se caracterizaban proxemia se ha extendido a las por acercarse al texto sujetándolo o relaciones de distancia, contacto e apropiarse del texto levantándolo. Entre interacción que las personas establecen los participantes de Ciclo Inicial con el mobiliario, la arquitectura y los destacaron las categorías de más objetos. En una extensión de esta idea, proximidad con el texto y en el Ciclo este trabajo descriptivo explora la Medio disminuyó significativamente el relación persona-texto en una muestra uso de la estrategia marcador (marcar la de 72 escolares que participan en una continuidad o los saltos de las líneas actividad de lectura en voz alta desde gráficas con los dedos), que vuelve a un pupitre, y analiza si la proxemia de recuperarse en los participantes de Ciclo los participantes guarda alguna Superior. Por último, se observan indicios relación con su etapa escolar, escuela o de que la escuela influye en la actitud nivel de competencia lectora. Se pidió proxémica del aprendiente.

a los participantes que leyeran un relato adecuado a su edad y se dibujó el modo en el que cada sujeto interaccionaba con el texto desde un punto de vista proxémico (ubicación y ángulo de los hombros, tronco, brazos, manos $\mathrm{y}$ cabeza). Después de un análisis inductivo de las ilustraciones, se concluyó que la muestra adoptaba hasta cuatro actitudes (categorías) 


\section{Introducción ${ }^{1}$}

La palabra proxémica (o proxemia) viene del latín proximus (que significa más cerca) y hace referencia, desde que el antropólogo estadounidense Edward. T. Hall la acuñara en 1963, a la manera en que los seres humanos estructuran y utilizan el espacio interpersonal. A mitades del siglo XX, numerosos estudios de etólogos dedicados a analizar cómo se comportaban los animales en el espacio; cómo lo distribuían y ocupaban de acuerdo con su rango o actividad, inspiraron, en el ámbito del paralenguaje, a hacer observaciones sobre la función, el uso y el significado de las distancias que mantenían las personas cuando se relacionaban. Desde entonces, el término proxemia ha aparecido de forma frecuente en los estudios que abordan la comunicación no verbal (ver, por ejemplo, algunos referentes como Knapp, 1982; Poyatos, 1994 o trabajos más recientes como el de Castro, 2013), si bien se trata de un término que sigue sin tener entrada en algunos de los diccionarios normativos, semasiológicos o de autoridades más destacados del español.

También en el ámbito de la arquitectura, el estudio de la proxemia se ha extendido al uso de las distancias entre las personas y los objetos y, sobre todo, en cómo influyen el mobiliario o las estructuras arquitectónicas en las relaciones interpersonales o las relaciones persona-objeto. Uno de los trabajos referentes en este ámbito fue el de Sommer (1969, p.249), donde pudo observarse cuántas veces (y por extensión, con qué calidad) llegaban a interactuar las personas que se encontraban alrededor de una mesa, dependiendo de su ubicación en ella. Esta línea de trabajo ha tenido una gran relevancia para el diseño de espacios privados cuyos usuarios necesitan mantener relaciones jerárquicas (por ejemplo, despachos de altos cargos); el diseño de museos que permitan establecer distintos tipos de relaciones con las obras; el diseño de espacio urbanos y sociales y, recientemente, también en el diseño, estudio y organización de un mobiliario escolar que facilite distintos tipos de relación con los elementos que intervienen en la actividad educativa (ver, por ejemplo, Cardellino y Araneda, 2014; Messi, 2015; Guamán, 2016 o Aguado, 2017).

En una extensión de esta idea, resulta interesante indagar en cuáles son las relaciones de distancia y de contacto que las personas establecen con los materiales escolares, más concretamente, en cuáles son las distintas proxemias que utilizan los escolares con los textos y qué calidad de lectura se desprende de cada una de ellas. Numerosos autores han dedicado sus estudios a los movimientos corporales de base psicomuscular que intervienen en la percepción visual o auditiva de aquello que se lee (véase, por ejemplo, Huey, 1908; Mcconkie, 1976; Crowder y Wagner, 1992 o Rayner y Sereno, 1994); así como a los patrones de movimiento de las manos en la lectura en braille (por ejemplo, Kusajima, 1974 o Rosa y Huertas, 1998). Pero no se encuentran, hasta Recio-Pineda $(2016)^{2}$, trabajos que indaguen en la interacción cuerpo-texto desde un sentido proxémico.

La variedad de contextos y objetivos de estudio en los trabajos sobre proxemia dispone a crear un desconcierto sobre cuáles son las variables que deben abordarse. A menudo, el contacto físico aparece como un aspecto que debe tratarse desde la kinésica (cfr. Birdwhistell, 1952). Y, sin embargo, no está claro que el contacto y la postura sean

1 Este artículo ha recibido financiación de ARCE (Universidad de Barcelona).

${ }^{2}$ Esta publicación explora, de forma preliminar, algunas de las cuestiones sobre las que se ahondará en este trabajo. 
variables independientes, así como tampoco la postura y la distancia. Por consiguiente, en este trabajo, se abordará una mirada que integre también información sobre si el lector establece contacto o no con el texto y de qué tipo, y sobre cuál es la orientación de sus hombros, brazos, manos, codos, tronco y cabeza cuando lee. A continuación, se realizará una breve revisión bibliográfica de las aportaciones metodológicas de Hall (1963) en este sentido.

En los estudios sobre proxemia, la variable descriptiva más utilizada ha sido la distancia. Sin embargo, para Hall (op.cit.), las relaciones espaciales debían comprenderse desde un complejo que tuviera en cuenta, también, factores como la orientación corporal y sus efectos sensitivos o la presencia o ausencia de contacto físico entre los sujetos, las personas y los objetos o la arquitectura. Este hecho puede verse reflejado en la definición de proxemia que ofrece el diccionario del Centro Virtual Cervantes (1997), según la cual, el lenguaje proxémico 'estudia las relaciones de proximidad-alejamiento, las posturas adoptadas y la existencia o ausencia de contacto físico entre las personas y los objetos durante la interacción'.

En su artículo A system for the notation of proxemic behavior (1963, p.1003), Hall propone 8 dimensiones sobre las que realizar un análisis sistemático del comportamiento proxémico; a saber: 1) identificadores posturales de sexo, 2) orientación sociofugalsociopetal, 3) factores kinésicos, 4) código táctico, 5) combinaciones de la retina, 6) código térmico, 7) código olfativo, y 8) escala de volumen de la voz.

- En la primera dimensión, identificadores posturales de sexo, conviene indicar el sexo de los interactuantes y si se encuentran de pie, sentados o acostados y si hay alguna diferencia notable en cuanto a sus edades, alturas o estatus.

- En lo que refiere a la segunda dimensión, la orientación sociofugal-sociopetal, el investigador debe describir la posición del hombro de una persona hacia la otra, siendo la situación cara a cara el máximo sociopetal o la postura que más favorece la interacción y de espaldas la orientación más sociofugal.

- Los factores kinésicos se refieren al cómo y dónde se tocan los interlocutores -con la cabeza o el tronco; con los antebrazos, los codos o las rodillas o con los brazos totalmente extendidos; dentro de una distancia mínima de contacto físico, justo fuera de una distancia mínima de contacto físico (distancia de agarre) o dentro o fuera de una distancia de alcance-.

- La cuarta dimensión, código táctico, describe el tipo de contacto físico: desde ningún contacto a contacto con caricia o con sujeción.

- Por su parte, la dimensión combinaciones de la retina hace referencia al grado de contacto visual que mantienen los interactuantes -fóveal, macular o periférico-. En general, cuanto más se alejen hacia la periferia, menos clara es la visión que conseguirán del otro.

- La dimensión código térmico describe la intensidad de la temperatura que se percibe desde las distintas distancias personales: calor conducido percibido, calor radiante percibido, calor probablemente percibido, calor no percibido,

- De un modo similar, el código olfativo describe la intensidad del olor que se percibe 
desde las distintas distancias: olor corporal detallado perceptible, etc.

- Por último, la escala de volumen de la voz codifica niveles de voz de muy suaves a muy altos, para ayudar a los participantes a calcular la distancia a la que quieren interactuar.

Más tarde, el mismo Hall publica un libro $(1968$, p.93) en el que describe 4 tipos de distancia entre dos profesionales adultos de clase media norteamericana que estaban realizando una entrevista: distancia íntima (menos de $45 \mathrm{~cm}$ ), la distancia personal o espacio casual-personal $(45-125 \mathrm{~cm})$, la distancia social o espacio social-consultivo $(1,20$ $2,10 \mathrm{~m}$ ) y la distancia pública $(3,50-7,50 \mathrm{~m})$, que permite adoptar una conducta de fuga o de protección-defensa en caso de sentirse incómodo. Cada uno de estos espacios proporciona una información distinta a los canales visuales, auditivos, olfativos, térmicos o tácticos, por lo que se deduce que las dimensiones se afectan e influyen mutuamente. Watson (1972, p.225) lo indicó del siguiente modo: "the total range of proxemic behavior is wide".

En el presente estudio, todos los participantes interactúan con el texto dentro de unos parámetros de intimidad, por lo que no se tendrán en cuenta los distintos tipos de distancia que ha descrito la bibliografía como un diferenciador entre las relaciones proxémicas que establecen los escolares con el texto. En vez de esto, se presentarán sus actitudes proxémicas en categorías complejas que arrojen información sobre las distintas dimensiones sobre las que analizar el comportamiento proxémico. Sin embargo, no se dará cuenta ni de los identificadores posturales de sexo, ni del código térmico, ni del código olfativo. En primer lugar, porque todos los participantes interactúan con un texto (y no con una persona sexuada) y lo hacen sentados en una silla del mobiliario escolar. En segundo lugar, porque las diferencias perceptivas entre el olor y la temperatura que se deducen de sus distintas estrategias proxémicas son mínimas y vienen determinadas por el campo de visión que consiguen. Por último, porque el volumen de la voz es en todos los casos "normal" (cfr. Hall, 1963, p.1013) y viene determinado por la distancia a la que se sitúa la grabadora (en el lateral izquierdo, a $40 \mathrm{~cm}$ del sujeto, aproximadamente).

Por otro lado, y tal y como ocurre en las interacciones sociales, cabría entender que la distancia entre el lector y el texto variaría en función de la edad, el sexo, el trasfondo cultural, el tema o el asunto, el ambiente para la interacción, las características del texto y el estado emocional del lector -que se intentó que fuera de tranquilidad y motivación en una conversación anterior a la lectura-. Erickson (1975) encontró que los movimientos espaciales entre los interlocutores podían marcar comienzos, finales y cambios de tema. De acuerdo con estos resultados, cabría esperar que los participantes realizaran cambios de postura y distancia en las diferentes fases de lectura y cada vez que reformularan sus hipótesis de lo que va a pasar en la comprensión en curso.

\section{Objetivos e hipótesis}

Este trabajo persigue tres objetivos:

1. Identificar las categorías y estrategias proxémicas de los lectores de Primaria.

2. Analizar la frecuencia en la que aparece cada una de las categorías 
proxémicas identificadas.

3. Explorar la frecuencia de aparición de las distintas categorías proxémicas en cada curso, nivel de competencia lectora y tradición escolar.

Para abordar el primer objetivo, se utilizó un método analítico-inductivo que permitiera extraer las distintas categorías proxémicas que se observaban en los participantes. Esto es: para categorizar y generalizar de cuántas maneras distintas interactuaban los hombros, brazos, manos, codos, tronco y cabeza de los participantes con el texto y, en definitiva, cuáles eran las diversas formas de relación de distancia y de contacto que los escolares establecían con el texto, en general. Una vez descritas estas categorías proxémicas, se analizó con qué frecuencia aparecían en toda la muestra, para detectar las más comunes. Después, se analizó la frecuencia de aparición de cada una de las categorías en los distintos estratos de edad, competencia lectora y escuela de procedencia para descubrir, de forma preliminar, si había tendencias que animaran a realizar estudios experimentales.

\section{Método}

El presente es un estudio descriptivo y exploratorio de las relaciones de distancia y de contacto que los escolares de Primaria establecen con el texto según su edad, nivel de competencia lectora y escuela, en un ejercicio de lectura en voz alta que se hace desde el pupitre.

\section{Participantes}

La muestra que participó en este estudio es heterogénea y está formada por 72 escolares de distintas edades, sexos, niveles de competencia lectora y centros de escolarización. Con todo, puede distribuirse en tres estratos más o menos homogéneos que contienen: 24 participantes de cada franja de edad o curso escolar $-2^{\circ}, 4^{\circ}$ y $6^{\circ}$ de Primaria, respectivamente, por ser estos los cursos que representan el final de cada Ciclo o etapa de Educación Básica-; 24 participantes de cada nivel de competencia lectora (8 en cada curso) -a saber: nivel de competencia lectora alta, nivel de competencia lectora media y nivel de competencia lectora baja, de acuerdo a las calificaciones y apreciaciones de sus maestros ${ }^{3}$ - y 36 participantes de cada escuela - pues, en este estudio, participan dos escuelas públicas y laicas de la misma comarca de Catalunya que siguen un enfoque ecléctico y mixto para la enseñanza y aprendizaje de las habilidades lectoras; en adelante, centro L y centro G (iniciales del nombre de la escuela)-.

A fin de controlar mejor las posibles variables distractoras en la selección de los estratos, se establecieron las siguientes condiciones:

- Todos los participantes debían haber sido escolarizados en el centro L o en el centro G desde Educación Infantil. En este punto, conviene recordar que uno de los objetivos de este estudio es explorar si el contexto-escuela es una variable que influya en las actitudes proxémicas de los lectores. Además, y, por otro lado, este criterio también permitía asegurar que todos los

\footnotetext{
${ }^{3}$ Debe entenderse, entonces, que la muestra fue seleccionada y tipificada junto al equipo de maestros y maestras de las distintas escuelas y de forma previa a la recogida de datos.
} 
participantes tuvieran un nivel de competencia suficiente en la lengua de lectura (en nuestro caso, el catalán ${ }^{4}$ ), ya que todos los participantes habrían entrado en contacto con esta lengua desde edades tempranas.

- Se excluyó del muestreo a los participantes que tuvieran un diagnóstico o necesidad educativa especial que pudiera afectar a sus habilidades lectoras tales como dislexia, ceguera, sordera, autismo o TDHA, entre otras.

El número de participantes de este estudio se decidió con criterios de conveniencia. Según el número de consentimientos informados que se recabaron de los padres para que los menores participaran de forma anónima y científica en el estudio, según la ley ${ }^{5}$, se seleccionó y estratificó a la muestra, de modo que hubiera el mismo número de informantes en cada curso, de cada escuela de procedencia, de cada sexo y de cada nivel de competencia lectora. El objetivo último de la selección era que la muestra fuera lo más amplia y heterogénea posible y que contuviera el mismo número de participantes en cada escuela y curso para poder triangular los resultados finales. La estratificación por niveles de competencia lectora de la muestra se realizó en abril de 2015 , para facilitar que las maestras tuvieran suficientes datos de los alumnos.

\section{Instrumentos/Material}

Para el desarrollo del estudio, se utilizaron tres textos narrativos (uno para cada franja de edad o ciclo escolar de la muestra), que tenían una cara de extensión y una letra adecuada a la edad de los participantes. Los textos fueron extraídos de libros de texto antiguos y se presentaron sin ilustraciones y plastificados.

Una libreta de campo sirvió para esbozar las posturas que adoptaban los distintos participantes al leer y la proxemia que establecían con el texto.

\section{Procedimiento}

El proceso de recolección de datos se llevó a cabo, en las dos escuelas, entre abril y junio de 2015. Debe tenerse en cuenta que los datos se recogieron participante por participante y que la investigadora tuvo que adaptarse a los ritmos, necesidades e posibilidades de la escuela.

Previo a la realización del estudio, se decidió, junto a los equipos docentes, que era conveniente que la investigadora pasara un día con los escolares de los distintos cursos y escuelas para presentarse como figura cercana y para explicar el motivo de su estancia en la escuela $^{6}$. Se explicó que realizaría una investigación sobre las distintas formas en las que se podía llegar a interpretar y reinterpretar una narración cuando se hablaba sobre ella. En ningún momento, sin embargo, se explicó que la investigadora tuviera interés en las relaciones de

\footnotetext{
${ }^{4}$ El catalán es una lengua oficial de la Comunidad de Catalunya. Se enseña en un contexto de inmersión total en la escuela para asegurar su permanencia (es una lengua minoritaria, respecto al castellano). Esto significa que los niños que están escolarizados en Catalunya reciben la educación de todas las materias (salvo las de otras lenguas) en catalán desde Educación infantil y hasta Educación Secundaria Obligatoria.

${ }^{5}$ En esta carta, se informaba de la posibilidad de autorizar la participación de los hijos en una actividad escolar de lectura que tenía fines científicos y para la cual se garantizaría la comodidad afectiva de los escolares y el tratamiento anónimo de sus datos.

${ }^{6}$ La recogida de datos se realizó durante días alternos de abril y mayo, según las necesidades y preferencias de los distintos maestros y maestras.
} 
proxemia que los lectores establecían con el texto para no condicionar las conductas de los participantes durante el estudio. Tras esta presentación, y una vez entregada la carta de consentimiento informado, se mantuvo una segunda reunión con los equipos docentes de las distintas escuelas para realizar la selección y tipificación de los estratos de la muestra y negociar un calendario.

Para la recogida de datos, y teniendo en cuenta otros objetivos de investigación que no se comentan en este trabajo, se pasó una prueba de lectura (para los participantes: 'una actividad') que tenía una duración aproximada ${ }^{7}$ de $30^{\prime}$ y que se realizaba, de forma individual y junto a la investigadora (un sujeto conocido para los participantes), en una sala especialmente habilitada para la ocasión -pequeña, con luz natural y ventilación, nivel de ruido medio-alto alrededor, estanterías llenas de material escolar y una mesa en el centro: grande, del mismo material y altura que los pupitres escolares y con 6-10 sillas alrededor-. La investigadora iba a buscar a los participantes (previa selección con el equipo docente) a sus clases y los acompañaba hasta la sala en una actitud comunicativa cercana. Una vez en la sala, la investigadora se situaba junto al participante, allí donde este hubiera decidido sentarse, con el cuerpo ligeramente orientado hacia él o ella para favorecer la interacción y continuar la conversación.

Tras la primera conversación informal con los participantes, se les mostraba el texto que iban a leer para que pudieran observarlo y realizar sus primeras hipótesis de lectura a partir del título. Después, se les explicaba que el objetivo de la actividad era leer y comprender el texto para después poder comentar el máximo de aspectos posibles sobre su trama. Para ello, era importante que los participantes estuvieran muy concentrados en su lectura y que volvieran atrás si en algún momento detectaban que se habían perdido. Una vez comprobado que los participantes entendían la instrucción, se les animaba a empezar a leer en voz alta.

Todos los datos - conversación informal, instrucciones, lectura en voz alta, comentario sobre diferentes aspectos de la trama con la investigadora- fueron grabados con una grabadora de audio. Además, se realizó un boceto de las actitudes corporales (proxemia de los participantes con el texto: distancia de la cabeza, tronco, brazos y manos y ángulo) en una libreta de campo y se anotó en qué momento del texto se producían, en cada caso. Después, se hizo un vaciado de las categorías proxémicas que se detectaron en los bocetos de la investigadora $\mathrm{y}$, al final, se estudió la frecuencia de aparición de cada una de las combinaciones proxémicas en cada curso, escuela y nivel de competencia lectora.

\section{Resultados}

En la Figura 1, se muestra un dibujo aproximado de las cuatro categorías proxémicas que se pudieron identificar y etiquetar en los bocetos de las notas de campo; a saber: 1) alejarse, 2) asomarse, 3) acercarse y 4) apropiarse al texto. Cada una de estas categorías aparece con dos o tres combinaciones o variedades; en adelante: estrategias -en diagonal, hacia atrás, sin apoyos...- que dan información sobre la posición de manos, brazos y tronco en el acto de lectura. En nuestro corpus de bocetos, se han detectado hasta 10 estrategias proxémicas distintas:

- $\quad$ Alejarse del texto en diagonal

${ }^{7}$ Cada participante destinó el tiempo que mejor se ajustaba a sus necesidades, ritmos de lectura, etc. 
- Alejarse del texto hacia atrás

- Asomarse al texto sin apoyos

- Asomarse al texto apoyando las manos y/o codos en la mesa [+/- marcador]

- Acercarse al texto rodeándolo

- Acercarse al texto sujetándolo o tocándolo en los márgenes [+/- marcador]

- Acercarse al texto tapándolo [+/- marcador]

- Apropiarse del texto cogiéndolo

- $\quad$ Apropiarse del texto levantándolo [+/-marcador]

- Apropiarse del texto acercándoselo

Como puede observarse, las distintas estrategias proporcionan información sobre diversas dimensiones de notación proxémica, en tanto que contemplan si la interacción con el texto se realiza cara a cara o de lado (como en alejarse en diagonal o alejarse hacia atrás), definen la distancia de la cabeza, el tronco, los codos y las manos y cómo se acercan o interactúan (apoyándose en la mesa, rodeando el texto, sujetando el texto, tapando el texto, cogiéndolo, levantándolo o acercándolo a los ojos) y permiten describir el campo de visión que se obtiene de cada actitud.

Debe notarse, además, que algunas estrategias como asomarse al texto apoyando las manos o codos en la mesa; acercarse al texto sujetándolo con los dedos; acercarse al texto tapándolo con las manos y/o brazos o apropiarse del texto levantándolo en posición vertical pueden ir acompañadas (o no) de un marcador. Se denomina estrategia marcador (cfr. Konstant 2003) ${ }^{8}$ a la acción de reseguir las líneas del texto con el dedo índice o marcar el principio y el final de cada línea con un dedo de cada mano para facilitar los saltos de la retina.

ALEJARSE DEL TEXTO

\footnotetext{
${ }^{8}$ Esta estrategia, de acuerdo con Konstant (2003), ayuda a aumentar la velocidad lectora y, en lo que concierne a nuestro estudio, aumenta el grado de actividad en la relación con el texto. 


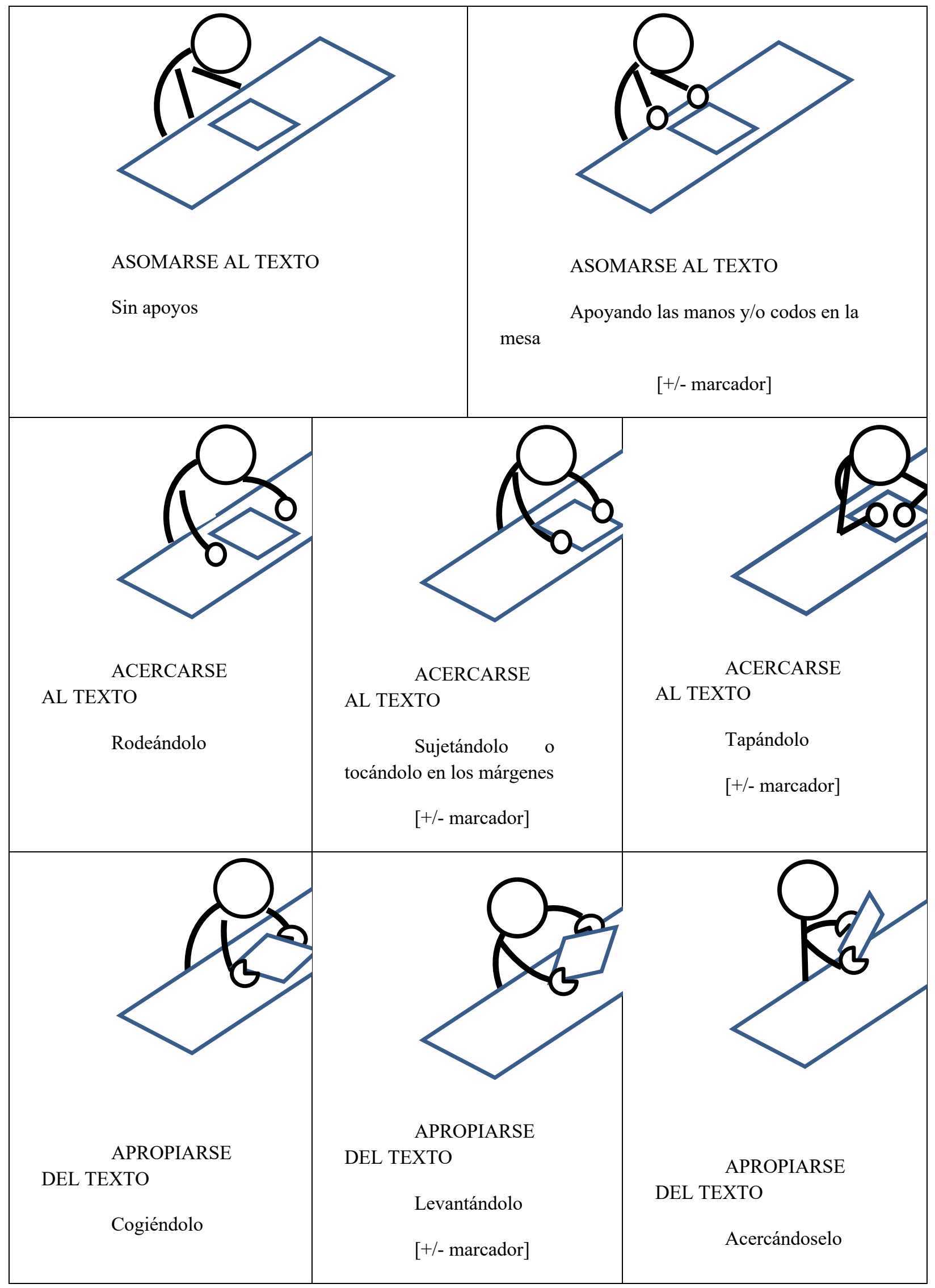

Figura 1. Imágenes de las estrategias proxémicas en los lectores de primaria

En las páginas que siguen, se muestra con qué frecuencia se registran cada una de las categorías proxémicas en los diferentes estratos de estudio de la muestra: curso, nivel de competencia lectora de los participantes y escuela de procedencia. Antes, sin embargo, se ofrece una descripción del comportamiento de los datos de forma genérica. 
A lo largo de las 72 pruebas de lectura se detectaron 86 cambios de postura, pues solo el $17 \%$ de los participantes modificó su actitud proxémica en el transcurso de la actividad lectora. A este respecto, las notas en el diario de campo explican que los lectores que más cambios proxémicos realizaron solían coincidir con los que menos se relacionaban con el texto...) en la fase anterior a la lectura (observarlo, tocarlo, hacerse una idea de la extensión, del título, del tema...).

Solo un $5 \%$ de los participantes adopta la categoría proxémica de máximo sociofugal. Esto es: contraria a favorecer la interacción con el texto y a participar de forma activa en la lectura. En los apartados que siguen, se comprobará que su aparición refiere solo a lectores con un nivel de competencia baja. También, que ocurre solo en uno de los centros (centro G).

En general, la categoría más usada por los escolares de Primaria es asomarse al texto: con y sin apoyos $(41 \%)$. Esta categoría se caracteriza por ofrecer un campo de visión amplio y una capacidad de focalización muy débil. De hecho, resulta fácil asociarla a la lectura de cuentos ilustrados, catálogos o revistas, en los que la imagen juega un texto relevante. Como se verá en las líneas que siguen, esta es la categoría que define a los lectores con una competencia lectora media o baja, especialmente en los ciclos Medio y Superior. Esta categoría viene muy pocas veces acompañada de una estrategia marcador $(2.32 \%)$. Por último, cabe decir que el $28 \%$ de los participantes que la usaron realizaron algún cambio de estrategia a lo largo de su lectura. Estos cambios, en su mayoría, se produjeron desde categorías proxémicas, a priori, más afines al éxito y a partir del primer tercio del fragmento leído. Este hecho podría ser un indicio de que la categoría asomarse aparece en situaciones de cansancio o pérdida en el proceso de comprensión.

Por su parte, las categorías acercarse y apropiarse aparecen en un orden de frecuencia similar; $29 \%$ y $25 \%$ respectivamente, y destacan sobre todo en los lectores con una competencia lectora media-alta. Por consiguiente, estas categorías serán sustancialmente diagnósticas de los buenos lectores de Ciclo Inicial. Se trata de las categorías que mayor oportunidad de focalizar en los fragmentos leídos ofrecen, y también, las que mejor permiten aplicar una estrategia marcador (reseguir el texto con los dedos o señalar los saltos de principio y final de línea); sobre todo en el caso de acercarse. En el $58 \%$ de los casos en los que la muestra se acerca el texto lo hace sujetándolo o tocándolo en los márgenes. Y en el $64 \%$ de los casos en los que la muestra se acerca al texto apropiándose de él, lo hace levantándolo. Las diferencias más significativas entre una y otra opción están en el campo de visión que se obtiene y en las posibilidades de aplicar un marcador que focalice en las líneas. La estrategia marcador, de hecho, apenas aparece asociada a la categoría apropiarse $(2.32 \%)$ y sí, en cambio, a la categoría acercarse (sobre todo sujetando o tocando el texto en los márgenes). En general, sin embargo, la estrategia marcador es un recurso poco usado por la muestra y destaca sobre todo en los lectores con una competencia lectora media-alta de Ciclo Inicial y en los lectores con una competencia media-baja de Ciclo Medio y de Ciclo Superior.

En el Gráfico 1, se muestran los porcentajes de aparición de cada categoría proxémica en cada uno de los cursos analizados. En él se refleja que la categoría que más aparece entre los lectores del Ciclo Inicial es acercarse, que aparece en el $36 \%$ de los casos registrados en este curso, seguida de la categoría apropiarse, que aparece con una frecuencia del $35 \%$. En el Ciclo Medio, la categoría que más destaca es asomarse, que 
aparece con una diferencia estadísticamente significativa: en un $52 \%$ de los casos. Por último, en el Ciclo Superior, las categorías que más destacan son asomarse, que aparece en el $46 \%$ de los casos registrados en este curso, y acercarse, que aparece en el $36 \%$.

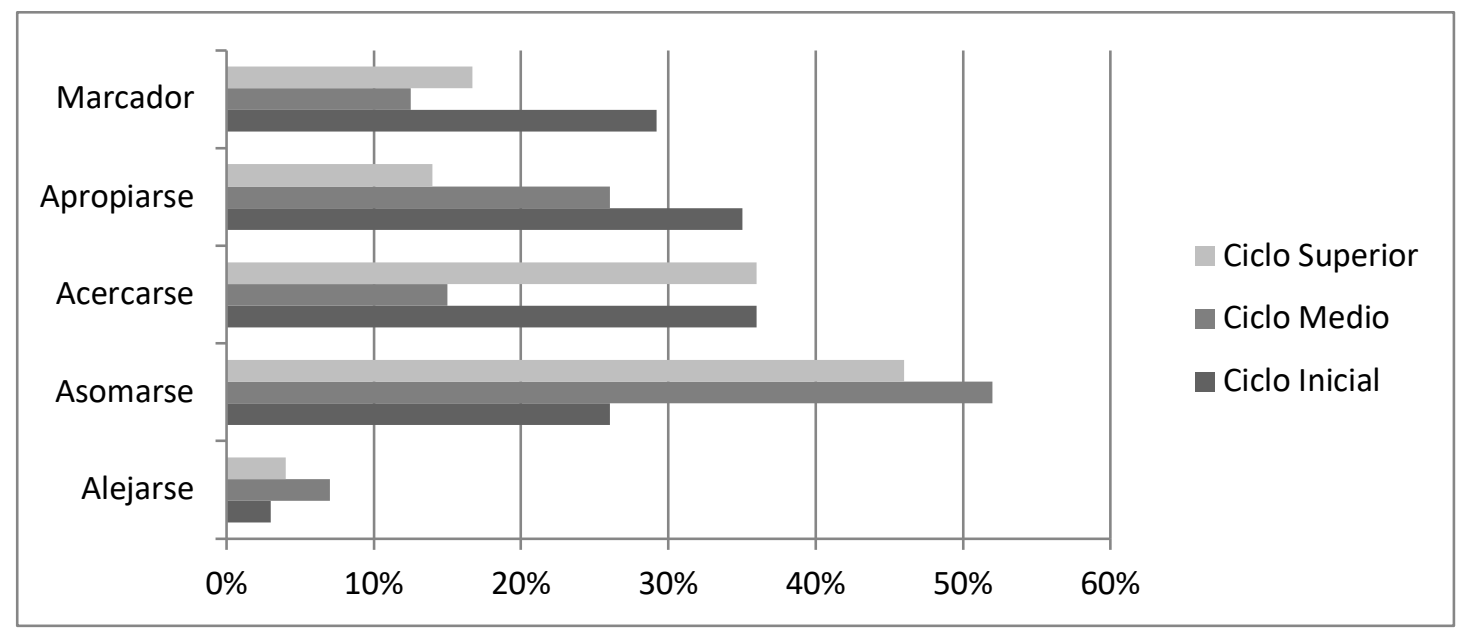

Gráfico 1. Relación entre categorías proxémicas y ciclo escolar

Los datos del Gráfico 1 muestran que los escolares de segundo curso de Primaria (Ciclo Inicial) mantienen las actitudes proxémicas que más favorecen el contacto con el texto: acercarse al texto sujetándolo y apropiarse del texto cogiéndolo o levantándolo. Este tipo de estrategias se acompaña en muchos casos del uso del dedo índice como marcador. Parece que en este Ciclo la tendencia es a relacionarse de forma activa con el texto. No hay que olvidar que, en esta etapa, las actividades de lectura reciben más atención que las demás actividades de lengua. Por consiguiente, se trabaja especialmente con la transmisión de hábitos de lectura, la relación de los escolares con los textos y el disfrute literario, acompañado de un constante refuerzo de las habilidades de descodificación y automatización de la lectura. De ahí que coincida que las estrategias más usadas en estos cursos sean las que más posibilidades de focalizar sobre un fragmento del texto ofrecen, y más contacto con el texto facilitan.

El acercamiento al texto se reduce en un $21 \%$ de ocasiones en los lectores de Ciclo Medio y se transforma en una tendencia a apropiarse, aunque menor que en el primer caso. Con todo, la muestra de participantes de Ciclo Medio es la que menos se relaciona de forma activa con el texto. Este grupo destaca en la categoría asomarse; categoría que también destaca entre los lectores con un nivel de competencia media-baja del Ciclo Superior. Este dato podría indicar la posibilidad de que asomarse sea una categoría que se encuentra en la zona de desarrollo próximo de los lectores que empiezan a combinar las habilidades de focalización con las habilidades de percepción del texto como unidad. Cuando esta estrategia se mantiene, resulta insuficiente para el Ciclo Superior, donde los lectores exitosos vuelven a acercarse al texto sujetándolo o apropiarse del texto levantándolo.

A modo de conclusión, los lectores de Ciclo Inicial son los que se relacionan de forma más activa: en las categorías más sociopetales, con más fuerza kinésica y táctica y con más posibilidades de focalizar sobre fragmentos leídos (acercarse y apropiarse), mientras que los lectores de Ciclo Medio y de Ciclo Superior son los que se relacionan de forma más pasiva; en categorías con poca fuerza kinésica y táctica y que dificultan las 
oportunidades de focalizar la vista en fragmentos de texto (alejarse, asomarse y acercarse al texto rodeándolo). A cambio, las categorías pasivas ofrecen más campo de visión.

A continuación, el Gráfico 2 explora las diferentes proxemias de los lectores de acuerdo con el nivel de competencia lectora que les atribuyen sus maestros. Los datos no permiten describir una tendencia en la frecuencia de aparición de las categorías proxémicas en los lectores que, de acuerdo con sus maestros, tienen un nivel de competencia lectora alta (A), si bien se puede afirmar que es altamente improbable que un lector con este grado de competencia lectora opte por alejarse del texto. Las tendencias de aparición de unas y otras categorías proxémicas son idénticas en el grupo de lectores con una competencia media (B) y en el grupo de lectores con una competencia lectora baja (C). Estos dos niveles de competencia destacan en la categoría asomarse y muestran menos predisposición que los lectores con un nivel de competencia lectora alta a apropiarse del texto. En este punto, puede ser conveniente estudiar la aparición de las distintas estrategias (y no categorías) en los distintos niveles de competencia.

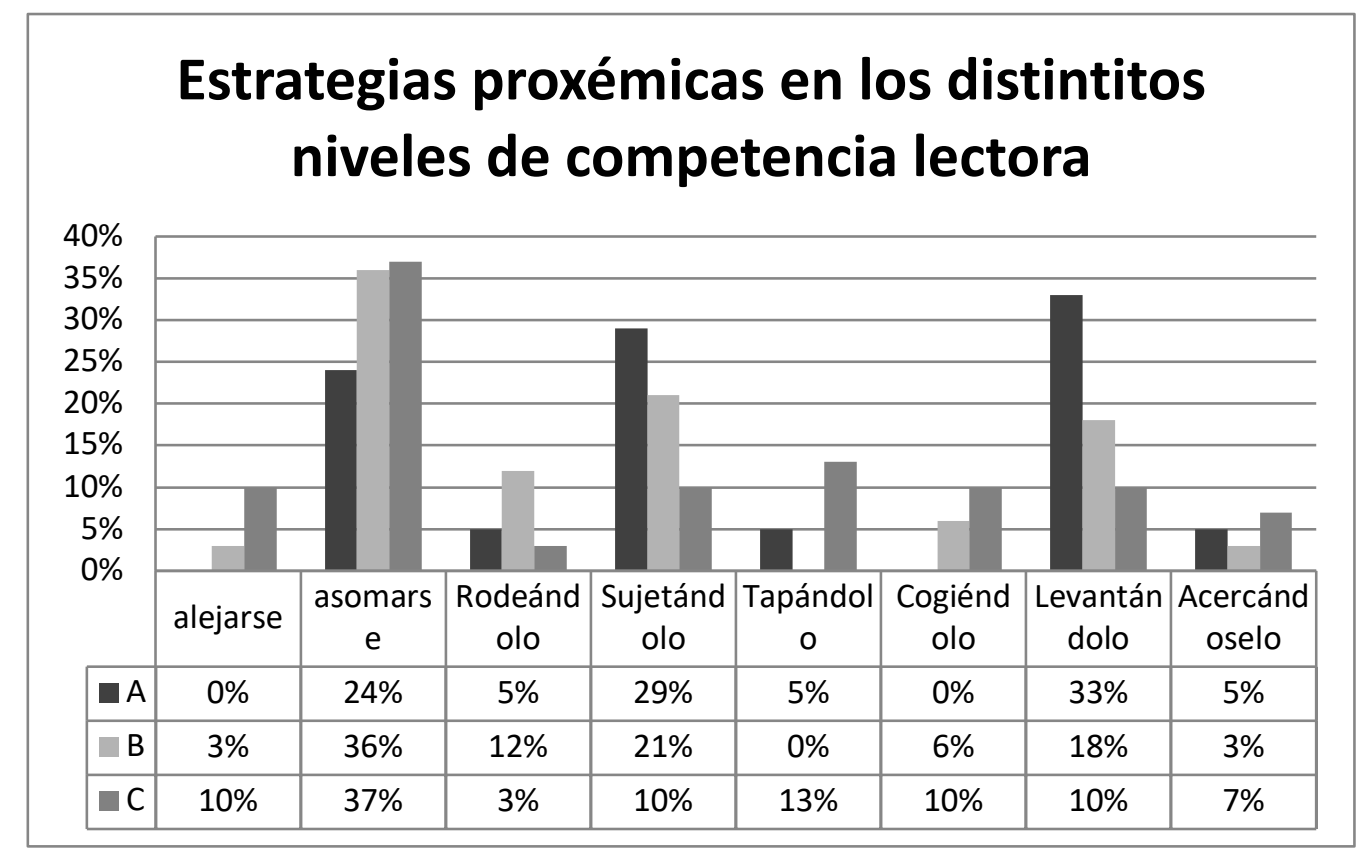

Gráfico 2. Estrategias proxémicas en los distintos niveles de competencia lectora

El Gráfico 2 arroja nuevas observaciones; por ejemplo, ningún lector con una competencia lectora alta se acerca al texto cogiéndolo, y muy pocos optan por acercarse rodeándolo o tapándolo. De hecho, acercarse al texto tapándolo parece ser una categoría exclusiva de los lectores con una competencia baja. A excepción de la categoría alejarse del texto y apropiarse del texto acercándoselo, que tal y como ya se ha comentado, son categorías poco comunes, todas las estrategias que descartan los buenos lectores (rodear el texto, taparlo, cogerlo) tienen una característica en común: ofrecen un campo de visión débil. A este respecto, conviene notar que, de acuerdo con Campayo (2009):

Para leer correctamente hay que abrir el campo de visión periférica y evitar a toda costa mirar en exclusiva lo que tenemos delante de nuestra nariz. Dicho de otro modo, hay que abrir nuestro campo de visión lateral tratando de emular una especie de ojo de pez (p.73). 
Y, en efecto, la estrategia preferida por los buenos lectores -acercarse al texto levantándolo- permite abrir el campo de visión. Las dudas aparecen con la estrategia acercarse sujetando el texto, que a priori no parece tener esta cualidad y sin embargo aparece de forma notoria en los lectores con una competencia lectora alta y los lectores con una competencia lectora media, y asomarse que, a pesar de ofrecer un campo de visión amplio, aparece de forma relevante en los lectores con una competencia lectora media y una competencia lectora baja.

Si los lectores con una competencia alta destacan con proxemias como sujetar o levantar el texto, los lectores con una competencia lectora baja destacan en proxemias como asomarse o acercarse tapando al texto (que, en cierto modo, es como asomarse muy cerca, con mayor foco y ningún campo de visión). Algo sí está claro: en las estrategias más usadas, los niveles de competencia lectora se distribuyen en un orden de apropiación: menos apropiación del texto, más usuarios con una competencia lectora baja; más apropiación del texto, más usuarios con una competencia lectora alta.

Para concluir este apartado, se estudia la frecuencia de aparición de cada categoría en cada uno de los centros de escolarización de la muestra. En el Gráfico 3, se observa en el centro L no se da ninguna estrategia proxémica de la categoría alejarse del texto y que esta categoría tampoco es frecuente en el centro G. En lo que se refiere a las estrategias de la categoría asomarse al texto, en la escuela $\mathrm{G}$ ambas estrategias son comunes; mientras que en la escuela L es mucho más frecuente la estrategia asomarse al texto sin apoyos. Las estrategias de la categoría acercarse al texto aparecen en ambos centros, si bien en el centro $\mathrm{G}$ destaca con la estrategia sujetándolo y tapándolo, frente a la estrategia rodeándolo, que destaca en el centro L. Finalmente, en la categoría apropiarse del texto, las estrategias cogiéndolo, levantándolo y acercándoselo aparecen de forma decreciente, con la notable excepción de la estrategia levantándolo, muy frecuente en la escuela G. Por consiguiente, parece que el contexto-escuela influye en la predisposición a usar unas y otras estrategias.

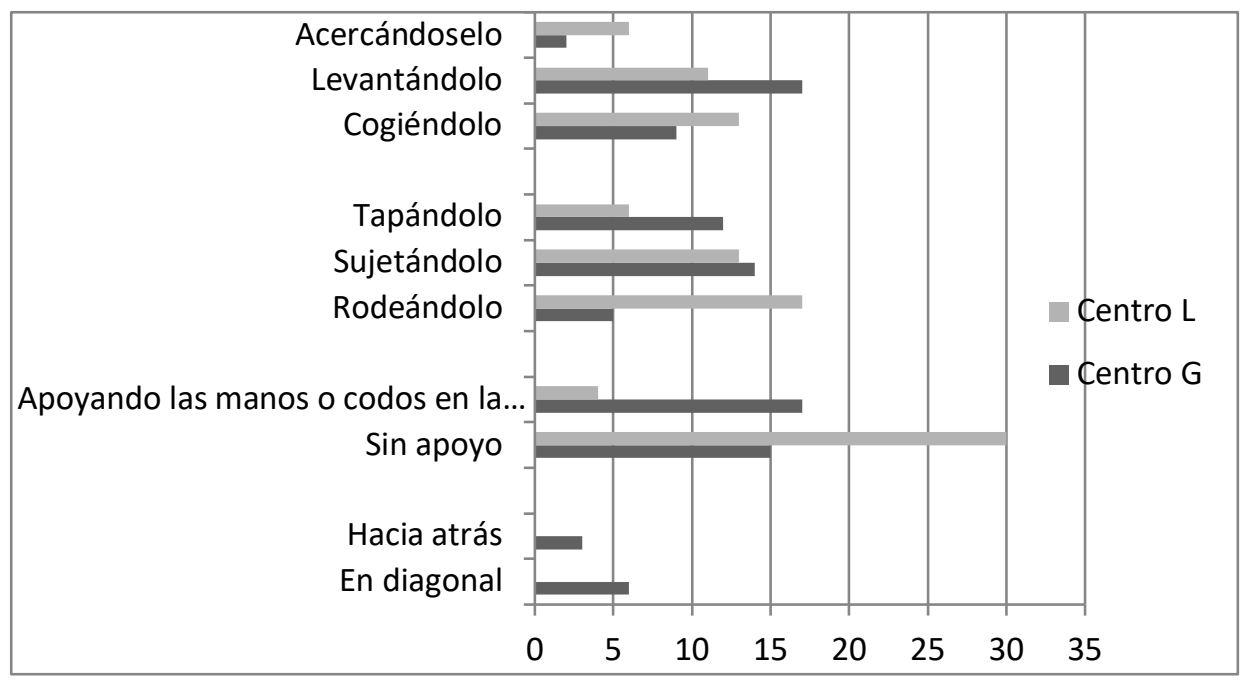

Gráfico 3. Relación entre categorías proxémicas y contexto escolar 


\section{Discusión y conclusiones}

La categoría 1, alejarse, sugiere, por un lado, aversión al texto -porque tiene muy poca fuerza de apropiación, de sociopetalidad y de actividad kinésica y táctica-. Por otro lado, esta categoría sugiere posibles problemas de vista, ya que cuando el lector se aleja del texto obtiene menos contacto fóveal. Alejarse conlleva nulas posibilidades de tener contacto con el texto; relacionarse con él. También, de focalizar sobre los fragmentos leídos. De hecho, podría compararse a la actitud de echarse atrás en la interacción entre personas, pero, como hemos visto, también podría indicar problemas de visión.

El campo de visión también es amplio en la categoría 2, asomarse, y esto permite poco foco en la retina, pero, a cambio, esta categoría aumenta el grado de relación de proximidad con el texto. La categoría 3, acercarse, puntúa con fuerza en todas las dimensiones, menos en el campo de visión. Por último, los lectores que 4) se apropian del texto; lo cogen, lo levantan o se lo acercan, estarían tomando posesión del objeto con el que interactúan y serían el ejemplo de mayor apropiación y capacidad de foco en la retina.

Las distintas estrategias aparecen, de hecho, ordenadas de acuerdo con la apropiación que los lectores mantienen con el texto -desde ninguna, en alejarse en diagonal, a una apropiación máxima, en apropiarse del texto acercándoselo-. A priori, podría entenderse que el grado de apropiación del texto es el resultado de combinar, de forma equilibrada, un buen nivel de sociopetalidad con el texto, un buen nivel de actividad kinésica y táctica, una amplitud en el campo de visión y una alta posibilidad de focalizar en las líneas del texto que se están leyendo. Sin embargo, conviene analizar las ventajas concretas de cada estrategia para ahondar más en detalle.

Si se ordenan las estrategias de acuerdo con el grado de cercanía que expresan los hombros con el texto, se podría considerar que las estrategias más sociofugales son: alejarse en diagonal, alejarse hacia atrás y apropiarse del texto cogiéndolo; sin embargo, las más sociopetales serían apropiarse del texto acercándoselo, apropiarse del texto levantándolo y acercarse al texto tapándolo. Llama la atención que apropiarse del texto cogiéndolo sea una estrategia sociofugal (que implica mayor ángulo de lejanía de los hombros y, por lo tanto, favorece poco la interacción con el texto) respecto a otras formas de apropiación o respecto a la estrategia de acercarse al texto tapándolo. Desde el punto de vista de la cercanía de los hombros con el objeto-texto, por tanto, la estrategia de apropiarse del texto cogiéndolo se parece mucho a asomarse al texto con apoyos.

Y muy en relación con la distancia a la que colocan los hombros del objeto-texto, se encuentra el factor kinésico (o grado de contacto físico que se establece con el texto). La estrategia de acercarse al texto tapándolo, que era la tercera en el orden de sociopetalidad, es la estrategia de mayor factor kinésico, junto a apropiarse del texto acercándoselo y apropiarse del texto levantándolo. Apropiarse del texto cogiéndolo estaría en el cuarto lugar de fuerza kinésica, mientras que las estrategias con menor factor kinésico serían las de alejarse y asomarse.

Este orden cambia cuando lo que se contempla es la fuerza con la que se establece el contacto, aunque se mantiene cierta coherencia con lo anterior. Las estrategias con un código táctico mayor son: apropiarse del texto acercándoselo, apropiarse del texto levantándolo y apropiarse del texto cogiéndolo, seguidas de acercarse al texto sujetándolo 
o tocándolo en los márgenes, y de acercarse al texto tapándolo. Las estrategias con menor código táctico son, de nuevo, alejarse y asomarse.

Por último, conviene analizar estas estrategias desde las combinaciones de la retina o grado de contacto visual que permiten con el texto. Si se atiende al campo de visión que puede obtenerse de cada estrategia, las estrategias que más destacan son alejarse y asomarse, y las que menos campo de visión ofrecen son las de la categoría acercarse. Las estrategias en la categoría de apropiarse, entonces, se caracterizan por un ofrecer un campo de visión medio. Por el contrario, si se analizan las estrategias desde la posibilidad de focalizar sobre ciertas partes del texto, las estrategias más idóneas son las de apropiarse y acercarse, y las que menos ayudan al lector en este aspecto son las de alejarse y asomarse.

En las líneas que siguen, se presenta un resumen de las conclusiones de este trabajo. Respecto a primer objetivo del trabajo, identificar las categorías y estrategias proxémicas de los lectores de Primaria, se concluye que:

Los lectores tienen múltiples estrategias proxémicas en la lectura. Esto es: no solo resiguen las líneas con los dedos o mueven los ojos y la boca, sino que también se relacionan con el cuerpo: alejándose del texto, asomándose al texto, acercándose al texto o apropiándose del texto con distintas estrategias -en diagonal, hacia atrás, sin apoyos, apoyando las manos y/o codos en la mesa, rodeando el texto, sujetándolo o tocándolo en los márgenes, tapándolo, cogiéndolo, levantándolo o acercándoselo.

Para describir la proxemia en la lectura, se pueden usar variables como la orientación de los hombros, tronco, cabeza y brazos del lector respecto al texto; la cantidad y la calidad de contacto físico que se produce con el texto y el ángulo de visión que se deduce de combinar las demás variables. Estas dimensiones fueron descritas por Hall (1963) para observar el comportamiento proxémico de dos personas que interactúan. Cabría ahondar en nuevas dimensiones que permitieran describir mejor la interacción con objetos y, en particular, textos.

En lo que refiere al segundo objetivo, analizar la frecuencia en la que aparece cada una de las categorías proxémicas en la muestra, concluimos que:

La mayoría de los lectores de Primaria se asoman al texto. Esta proxemia ofrece un campo de visión muy amplio y una capacidad de focalización muy débil. En el corpus, esta proxemia aparece asociada a los lectores con una competencia lectora media-baja y a situaciones de cansancio o pérdida en el proceso de comprensión. Es muy raro encontrar la proxemia alejarse del texto. Por su parte, las proxemias de acercarse o apropiarse aparecen en el 25-30\% de los casos. El $16 \%$ de los casos descritos utiliza en algún momento (o durante toda la actividad de lectura) la estrategia marcador. Esta estrategia suele aparecer en los lectores que sujetan el texto y, sobre todo, entre los alumnos y alumnas de Ciclo Inicial. Convendría valorar si los escolares que la utilizan obtienen o no mejores resultados de comprensión. La bibliografía indica que esta estrategia puede ayudar a desarrollar una mayor fluidez.

Por último, al relacionar las categorías proxémicas con el curso, nivel de competencia lectora y tradición escolar, se observa que:

En cuanto a las proxemias detectadas en los distintos cursos, los participantes que 
realizan más cambios de postura durante la realización de las pruebas pertenecen al Ciclo Inicial y coinciden con los participantes que menos se relacionaron con el texto (mirarlo, observarlo, tocarlo) antes de empezar a leer. También se concluye que en el Ciclo Inicial destacan las categorías acercarse al texto y apropiarse del texto, sobre todo sujetándolo y aplicando estrategia marcador o levantándolo; esto es, las categorías de más proximidad con el texto (fuerza sociopetal, fuerza kinésica, fuerza táctica y foco de visión). En el Ciclo Medio, destaca la estrategia asomarse al texto y apropiarse del texto levantándolo y disminuye significativamente el uso de la estrategia marcador. Por último, en el Ciclo Superior, destacan las estrategias asomarse al texto y acercarse al texto sujetándolo, lo que vuelve a posibilitar el uso de estrategias marcador.

Si se relacionan las distintas proxemias con el nivel de competencia lectora de los escolares, se observa que las categorías que más se describen en Ciclo Inicial coinciden con las categorías que más destacan en los lectores con una competencia lectora alta. Sin embargo, las categorías que más aparecen en el Ciclo Medio y Superior destacan entre los lectores con un nivel de competencia media-baja. Esto se debe, sobre todo, al peso que recibe la estrategia asomarse en los dos últimos Ciclos.

Ningún lector con competencia lectora alta opta por alejarse del texto ni por apropiarse del él cogiéndolo. Además, es infrecuente que se acerque para rodear el texto, taparlo o que se apropie del texto acercándoselo. Este tipo de lector prefiere apropiarse del texto levantándolo o acercarse a él para sujetarlo. Levantar el texto es una de las estrategias proxémicas que más fuerza de apropiación, sociopetal, kinésica, táctica y de foco tiene. Y a diferencia de acercarse y sujetarlo, permite un mayor campo de visión. Por su parte, la estrategia acercarse al texto y sujetarlo puntúa con valores medios en todas las dimensiones proxémicas de estudio, así que parece ser muy útil y equilibrada para el lector. En el otro extremo, los lectores con una competencia lectora baja destacan en la categoría asomarse, si bien, como hemos visto, esta es una categoría que abunda en todos los niveles. También el grupo de lectores con una competencia lectora baja acostumbra a acercarse al texto tapándolo. Por último, es en este grupo donde se registran los casos de alejarse del texto.

En resumen, las estrategias más exitosas, o que mejor describen a los lectores con una competencia lectora alta, son acercarse al texto sujetándolo y apropiarse del texto levantándolo. Por el contrario, alejarse del texto, apropiarse del texto cogiéndolo y acercarse al texto tapándolo son estrategias ineficaces. La categoría más común; asomarse al texto, aparece en diversos tipos de lectores de diversos cursos, pero destaca sobre todo en los lectores con una competencia lectora media-baja. Se podría decir que cuánto más apropiativa es la categoría proxémica, más común es encontrarla entre los lectores de competencia lectora alta y a la inversa: cuanto menos apropiativa es la categoría, más común es encontrarla entre los lectores de competencia lectora baja. De hecho, los lectores con una competencia lectora baja del Ciclo Superior no se apropian del texto. Por último, conviene notar que las estrategias acercarse rodeando el texto y apropiarse del texto acercándoselo no distinguen lectores con mayor o menor nivel de competencia lectora, pero sí lectores de uno y otro curso escolar: Superior e Inicial respectivamente

Por último, en cuanto a la relación entre actitudes proxémicas y cultura escolar, se observan indicios del contexto afectivo que envuelve la actividad de lectura, así como las personas que actúan como modelos lectores, podrían influir en la actitud proxémica del 
aprendiente. De hecho, se sabe que parte importante del aprendizaje inicial de la lectura constituye un juego de imitación del acto de leer (basado en modelos). En este juego inicial, el neolector asume que leer incluye coger algún material escrito y mirarlo; esto es: lo primero en lo que se fija es en la actitud corporal y la interacción persona-texto. Tal vez, este juego continúe también en Primaria, donde el escolar se relaciona, paulatinamente, con nuevos géneros, formatos, estrategias y voces lectoras. En el centro $\mathrm{G}$, es común encontrar lectores que se asoman al texto con apoyos y lectores que se apropian de él levantándolo. Solo en este centro se registran casos de alejarse del texto. En el centro L, destacan más los lectores que se asoman sin apoyos y que se acercan al texto rodeándolo.

\section{Referencias}

Aguado, J. C. (2017). Análisis etnológico de la proxemia de un grupo otomí migrante a la Ciudad de México. Alteridades, 27(53), 83-94. Recuperado el 20 de agosto, 2018 de: $\quad$ http://www.scielo.org.mx/scielo.php?script=sci_arttext\&pid=S0188$\underline{70172017000100083 \& \operatorname{lng}=\mathrm{es} \& \mathrm{nrm}=\mathrm{iso}}$

Birdwhistell, R. L. (1952). Introduction to kinesics. Louisville: University of Louisville.

Campayo, R. (2009). Curso definitivo de lectura rápida: Método Ramón Campayo. Madrid: Edaf.

Cardellino, P., y Araneda, C. (2014). Mapeo Vivencial del Fenómeno EnseñanzaAprendizaje en el Espacio de Aula Escolar-El Caso de Uruguay. Blucher Design Proceedings, 1(8), 144-147.

Castro, M. D. M. (2013). La cortesía: códigos verbales y no verbales en la comunicación interpersonal/Courtesy: verbal and non-verbal codes at interpersonal communication. Historia Y Comunicación Social, 18, 365-375. Recuperado el 20 de agosto, $2018 \quad$ de: http://revistas.ucm.es/index.php/HICS/article/view/44248/41811

Centro Virtual Cervantes (1997). "Proxémica" [en línea]. Diccionario De Términos clave de ELE. Madrid: Instituto Cervantes. Recuperado el 8 de enero, 2018 de: http://cvc.cervantes.es/ensenanza/biblioteca_ele/diccio_ele/diccionario/proxemic a.htm

Crowder, R. y Wagner, R. (1992). The psychology of reading: An introduction (2nd ed.). New York, US: Oxford University Press.

Erickson, F. (1975). One function of proxemic shifts in face-to-face interaction. En Kendon A., Harris, R. H. y Ritchie Key, M. (Eds.). Organization of behaviour in face-to-face interactions. (pp. 115-187). Chicago: Aldine.

Guamán, A. D. (2016). Recuperación de hábitos y tradiciones de vida a través de la organización del espacio urbano en las ciudades. (Trabajo de graduación para optar al Título de Arquitecto). UCE, Quito. Recuperado el 20 de agosto, 2018 de: http://www.dspace.uce.edu.ec/handle/25000/9514

Hall, E. T. (1963). A system for the notation of proxemic behavior. American Anthropologist, 65(5), 1003-1026. Recuperado el 11 de enero, 2018 de: http://onlinelibrary.wiley.com/doi/10.1525/aa.1963.65.5.02a00020/abstract

Hall, E. T. (1968). Proxemics. Current Anthropology, 9(2/3), 83-108. Recuperado el 10 
de enero, 2018 de: http://www.journals.uchicago.edu/doi/abs/10.1086/200975

Huey, E. B. (1908). The psychology and pedagogy of reading. Cambridge, MA: M.I.T Press.

Knapp, M. (1982). La comunicación no verbal: El cuerpo y el entorno. Barcelona: Paidós.

Konstant, T. (2003). Técnicas de lectura rápida para leer más rápido y sacar más provecho de la lectura. Barcelona: Amat editorial.

Kusajima, T. (1974). Visual reading and braille reading: An experimental investigation of the physiology and psychology of visual and tactual reading. New York, US: American foundation for the Blind.

Mcconkie, G. (1976). The use of eye-movement data in determining perceptual span in reading. En Monty, R.A. y Senders, J. W. (Eds.). Eye movements and psychological processes. Hillsdale, NJ: Lawrence Erlbaum.

Messi, V. (2015). Proxemia: análisis sociotécnico de una obra de arte electrónico. En: Alsina, P. y Rodríguez, A. (Coord). «Art Matters II». Artnodes, 16, 34-42. UOC. Recuperado el 20 de agosto, 2018 de: http://journals.uoc.edu/ojs/index.php/artnodes/article/view/n16-messi/n16-messipdf-es

Poyatos, F. (1994). La comunicación no verbal II. Paralenguaje, kinésica e interacción. Madrid: Istmo.

Rayner, K. y Sereno, S. (1994). Regressive eye movements and sentence parsing: On the use of regression-contingent analyses. Memory \& Cognition, 22, 281-285. Recuperado el 10 de enero, 2018 de: https://link.springer.com/article/10.3758/BF03200855

Recio-Pineda, S. (2016). Los lectores también tienen cuerpo. En A.E. Díez Mediavilla, V. Brotons Rico, D. Escandell, J. Rovira-Collado (coord.), Aprendizajes plurilingües y literarios: Nuevos enfoques didácticos (p. 751-760).

Rosa, A. y Huertas, J.A. (1998). Peculiaridades de la lectura táctil del braille: Un estudio empírico. Infancia y Aprendizaje, 41, 79-94. https://doi.org/10.1080/02103702.1988.10822191

Sommer, R. (1969). Personal space: The Behavioral Basis of Design. Englewood Cliffs, N.J: Prentice-Hall (Spectrum)

Watson, O. M. (1972). Proxemics as non-verbal communication. En Ghosh, S.K. (Ed.). Man, language and society: Contributions to the sociology of language. (pp 224231). The Hague: Mouton. 


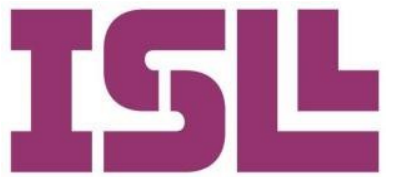

Number 11

2019 June

\title{
Proxemics in reading: how elementary schoolchildren approach the text according to their age, context and reading competence level
}

\author{
Sara Recio-Pineda \\ University of Barcelona \\ https://orcid.org/0000-0002-7823-3504
}

Received: 11-01-2019

Accepted: 25-06-2019

Pag. 19 to 36

\section{Keywords}

proxemics, reading, primary school, postural code, reading competence

\begin{abstract}
:
Some readers distance themselves concluded that 4 proxemic categories with from the text they are reading, others different versions could be extracted from look at it and approach it from their the corpus. We found that the majority of seats, others come closer covering the readers chose to lean out the text, looking text, touching it or laying their arms in at it, although readers who according to the surrounding spaces, and many their teachers had a higher level of reading other readers seize the text making it competence would approach the text by theirs, placing it closer to them. In the holding it or either they would appropriate past recent years, the study of the text by raising it. The categories that proxemics includes the relationships of showed a better proximity to the text stood distance, contact and interaction that out among second grade participants. The people establish with furniture, use of a finger-pacer strategy experienced architecture and objects. As an a fall among fourth grade readers, but we extension of this idea, this study aims observed an increase in the use of this to describe and explore how a sample strategy again among sixth grade of 72 schoolers interacts with the text schoolers. Finally, we found evidence that in the course of an individual activity the school context influences the proxemic that they do from a desk at school. We behavior of learners.
\end{abstract}

also analyze the connection between the proxemics of the participants and their age, school and reading competence. We asked participants to read a short story that was appropriate to their age and we captured a drawing of their proxemics in relation to the text (location and angle of the shoulders, trunk, arms, hands and head). After analyzing the different drawings, we 


\section{Introduction ${ }^{9}$}

The word proxemics (or proxemia) comes from the Latin proximus (which means closer) and makes reference, since the American anthropologist Edward. T. Hall coined it in 1963, to the way human beings, structure and use interpersonal space. In the midtwentieth century, numerous studies of ethologists were dedicated to analyzing how animals behaved in space, including how they distributed and occupied it according to their rank or activity. These studies inspired some scholars, in the field of parallel language, to make observations about the function, use and meaning of the distances that people maintained when they related to each other. Since then, the term proxemics has appeared frequently in studies that address nonverbal communication (see, for example, references such as Knapp, 1982; Poyatos, 1994 or more recent works such as Castro, 2013), although it is a term that still has no entry in some of the normative, semasiological or leading Spanish dictionaries.

In addition, in the field of architecture, the study of proxemics has extended to the use of distances between people and objects and, above all, how furniture or architectural structures influence interpersonal relationships or personal relationships with an object. One of the reference works in this area is that of Sommer (1969, p.249), where it was observed how many times (and by extension, with what quality) the people who were around a table came to interact, depending on their location. This line of work has had great relevance for the design of private spaces whose users need to maintain hierarchical relationships (for example, offices of high positions); the design of museums that allow establishment of different types of relationships with the works; the design of urban and social spaces; and, recently, also in the design, study and organization of school furniture that facilitates different types of relationships with the elements involved in the educational activity (see, for example, Cardellino \& Araneda, 2014; Messi, 2015; Guamán, 2016; Aguado, 2017).

In an extension of this idea, it is interesting to inquire into what the distance and contact relationships are that people establish with school materials, more specifically, what are the different proxemics patterns observed in schoolchildren when they try to read a text and what quality of reading follows from each of them. Numerous authors have dedicated their studies to psycho-muscular-based body movements that intervene in the visual or auditory perception of what is read (see, for example, Huey, 1908; Mcconkie, 1976; Crowder \& Wagner, 1992 or Rayner \& Sereno, 1994); as well as the patterns of hand movement in braille reading (for example, Kusajima, 1974 or Rosa \& Huertas, 1998). But, until Recio-Pineda (2016), works that investigate human proxemics in a reading activity are not found.

The variety of contexts and objectives of study in the work on proxemics has created a confusion about what variables must be addressed. Often, physical contact appears as an aspect that must be treated from the kinesics perspective (cf. Birdwhistell, 1952). And yet, it is not clear that contact and posture are independent variables, nor is posture and distance. Therefore, in this work, a look will be addressed that also integrates information about whether or not readers make contact with the text and of what type,

\footnotetext{
${ }^{9}$ This paper has received funding from the ARCE (University of Barcelona)
} 
and what the orientation is of their shoulders, arms, hands, elbows, trunk and head when he reads. Next, a brief bibliographic review of Hall's methodological contributions (1963) will be carried out in this regard.

In studies on proxemics, the most used descriptive variable has been distance. However, for Hall (op.cit.), spatial relationships should be understood from a complex perspective that also takes into account factors such as body orientation and its sensory effects or the presence or absence of physical contact between subjects, people and objects or architecture. This fact can be seen reflected in the definition of proxemics offered by the dictionary of the Cervantes Virtual Center (1997), according to which, the proxemic language "studies the proximity-distance relations, the adopted positions and the existence or absence of physical contact between people and objects during interaction".

In his article, "A system for the notation of proxemic behavior" (1963, p. 1003), Hall proposes eight dimensions on which to perform a systematic analysis of the proxemic behavior; namely: 1) postural-sex identifiers, 2) sociofugal-sociopetal orientation, 3) kinaesthetic factors, 4) touch code, 5) retinal combination, 6) thermal code, 7) olfaction code, 8) voice loudness scale.

- In the first dimension, postural-sex identifiers, it is advisable to indicate the sex of the interacting parties and whether they are standing, sitting or lying down and if there is any notable difference in their ages, heights or status.

- Regarding the second dimension, the sociofugal-sociopetal orientation, the researcher must describe the position of one person's shoulder towards the other, with the face-to-face situation being the maximum sociopetal or the position that most favors interaction, and back-facing as the most sociofugal orientation.

- $\quad$ Kinaesthetic factors refer to how and where the interlocutors touch each other with the head or trunk; with the forearms, elbows or knees or with the arms fully extended; within a minimum distance of physical contact, just outside a minimum distance of physical contact (grip distance) or within or outside a range of reach.

- The fourth dimension, touch code, describes the type of physical contact: from any contact to contact with caress or holding.

- $\quad$ The retinal combinations dimension refers to the degree of visual contact that the interactives maintain -foveal, macular or peripheral-. In general, the further they move towards the periphery, the less clear the vision they will have of the other.

- $\quad$ The thermal code dimension describes the intensity of the temperature that is perceived from different personal distances: perceived conducted heat, perceived radiant heat, probably perceived heat, unperceived heat.

- In a similar way, the olfaction code describes the intensity of the smell that is perceived from the different distances: perceptible detailed body odor, etc.

- $\quad$ Finally, the voice loudness scale encodes voice levels from very soft to very high, in order to help participants, calculate the distance at which they want to interact. 
Later, the same Hall published a book (1968, p. 93) in which he describes four types of distance between two adult middle-class American professionals who were conducting an interview: intimate distance (less than $45 \mathrm{~cm}$ ), personal distance or casualpersonal space $(45-125 \mathrm{~cm})$, social distance or social-consultative space $(1.20-2.10 \mathrm{~m})$ and public distance $(3.50-7.50 \mathrm{~m})$, which allows them to adopt a flight behavior or of protection-defense in case of feeling uncomfortable. Each of these spaces provides different information to visual, auditory, olfactory, thermal or tactical channels, so it follows that the dimensions affect and influence each other. Watson (1972, p. 225) indicated as follows: "the total range of proxemic behavior is wide".

In the present study, all participants interact with the text within privacy parameters, so the different types of distance that the literature has described as a differentiator will not be taken into account text. Instead, their proxemic behaviors will be presented in complex categories that yield information about the different dimensions in which to analyze proxemic behavior. However, you will not notice the postural sex identifiers, the thermal code or the olfactory code. First, because all participants interact with a text (and not with a sexed person) and they do so, sitting in a chair that is part of the school furniture. Secondly, because the perceptual differences between the smell and the temperature that are deduced from their different proxemic strategies are minimal and are determined by the field of vision they achieve. Finally, the volume of the voice is, in all cases, "normal" (cf. Hall, 1963, p.1013) and is determined by the distance at which the recorder is located (on the left side, approximately $40 \mathrm{~cm}$ from the subject,).

On the other hand, and as is the case in social interactions, it should be understood that the distance between the reader and the text would vary depending on age, sex, cultural background, theme or subject, the environment for interaction, the characteristics of the text and the emotional state of the reader -which was intended to be of tranquility and motivation in a conversation prior to reading-. Erickson (1975) found that spatial movements between the interlocutors could mark beginnings, endings and changes of subject. According to these results, it would be expected that the participants made changes in posture and distance in the different reading phases and each time they rephrased their hypotheses of what will happen in the current understanding.

\section{Objectives and hypotheses}

This work has three objectives:

1. Identify the proxemic categories and strategies of primary school readers.

2. Analyze the frequency at which each of the identified proxemic categories appears.

3. Explore the frequency of occurrence of the different proxemic categories in each course, reading proficiency level and school tradition.

To address the first objective, an analytical-inductive method was used to extract the different proxemic categories observed in the participants. This is: to categorize and generalize how many different ways the participants' shoulders, arms, hands, elbows, trunk and head interacted with the text and, in short, what the different forms of distance and contact relationships were that schoolchildren set with the text, in general. Once these 
proxemic categories were described, it was analyzed how often they appeared throughout the sample, in order to detect the most common ones. Then, the frequency of occurrence of each of the categories in the different age strata, reading competence and school of origin were analyzed to discover, in a preliminary way, if there were trends that would encourage experimental studies.

\section{Methodology}

This is a descriptive and exploratory study of the distance and contact relationships that primary school students establish with the text according to their age, reading proficiency level and school, in an exercise of reading aloud that is done from the desk.

\section{Participants}

The sample that participated in this study is heterogeneous and consists of 72 schoolchildren of different ages, sexes, reading proficiency levels and school centers. However, it can be distributed into three more or less homogeneous strata that contain: 24 participants of each age group or school year $-2 \mathrm{nd}$, 4th and 6th grade, respectively, since these are the courses that represent the end of each Cycle or stage of basic education-; 24 participants of each reading proficiency level (eight in each course) namely: high reading proficiency level, medium reading proficiency level and low reading proficiency level, according to the qualifications and assessments of their teachers ${ }^{10}$ - and 36 participants from each school -which included, in this study, two public and secular schools from the same region of Catalonia, each following an eclectic and mixed approach to teaching and learning reading skills; hereinafter, center $\mathrm{L}$ and center $\mathrm{G}$ (initials of the name of the school)-.

In order to better control for the possible distracting variables in the selection of strata, the following conditions were established:

- All participants should have been enrolled in center L or center G from Early Childhood Education. At this point, it should be remembered that one of the objectives of this study is to explore whether the school context is a variable that influences the proxemic behaviors of readers. In addition, and on the other hand, this criterion also allowed us to ensure that all participants had a sufficient level of proficiency in the reading language (in our case, Catalan ${ }^{11}$ ), since all participants would have come into contact with this language from an early age.

- Participants who had a diagnosis or special educational need that could affect their reading skills such as dyslexia, blindness, deafness, autism or TDHA, among others, were excluded from sampling.

\footnotetext{
${ }^{10}$ It should be understood, then, that the sample was selected and typified together with the team of teachers of the different schools and prior to data collection.

${ }^{11}$ Catalan is an official language of the Community of Catalonia. It is taught in a context of total immersion in the school to ensure its permanence (it is a minority language, with respect to Spanish). This means that children who are enrolled in Catalonia receive education in all subjects (except those of other languages) in Catalan from Early Childhood Education and to Compulsory Secondary Education.
} 
The number of participants in this study was decided with convenience criteria. According to the number of informed consents that were collected from the parents so that the minors participated anonymously and scientifically in the study, according to the law $^{12}$, the sample was selected and stratified, so that there were the same number of informants in each course, of each school of origin, of each sex and of each level of reading competence. The ultimate goal of the selection was that the sample be as wide and heterogeneous as possible and that it contained the same number of participants in each school and course in order to triangulate the final results. The stratification by reading proficiency levels of the sample was carried out in April 2015 in order to ensure that the teachers had sufficient data about the students.

\section{Materials/ Instruments}

For the development of the study, three narrative texts were used (one for each age group or Cycle of the sample). The texts had an extension of one page and a font size appropriate to the age of the participants. The texts were extracted from old textbooks and were presented without any illustrations and plasticized.

A field book served to capture a drawing of their positions and the proxemics they established with the text when they were reading.

\section{Procedure}

The data collection process was carried out, in the two schools, between April and June 2015. It should be taken into account that the data was collected participant-byparticipant and that the researcher had to adapt to the rhythms, needs and possibilities of the school.

Before carrying out the study, it was decided, together with the teaching teams, that it was convenient for the researcher to spend a day with the schoolchildren of the different courses and schools in order to present themselves as a close figure and to explain the reason for their stay at the school ${ }^{13}$. It was explained that he would conduct an investigation into the different ways in which a narrative could be interpreted and reinterpreted when talking about it. At no time, however, was it explained that the researcher had an interest in the relationships of proxemics that the readers established with the text so as not to condition the behaviors of the participants during the study. After this presentation, and once the letter of informed consent was delivered, a second meeting was held with the teaching teams of the different schools to carry out the selection and typing of the strata of the sample and to negotiate a calendar.

For the collection of data, and taking into account other research objectives that are not commented on in this work, a reading test was passed (for the participants: "an activity") that had an approximate duration ${ }^{14}$ of 30 seconds and that was carried out, individually and together with the researcher (a subject known to the participants), in a

\footnotetext{
${ }^{12}$ In this letter, the possibility of authorizing the participation of children in a school reading activity that had scientific purposes and for which the emotional comfort of schoolchildren and the anonymous treatment of their data would be guaranteed was informed.

${ }^{13}$ Data collection was carried out during alternate days of April and May, according to the needs and preferences of the different teachers.

${ }^{14}$ Each participant set aside the time that best suited their needs, reading rhythms, etc.
} 
room specially enabled for the occasion -a small space, with natural light and ventilation, medium-high noise level around, shelves full of school supplies and a center table that was large, of the same material and height as school desks and with 6-10 chairs around it-. The researcher was going to collect the participants (previous selection with the teaching team) from their classes and accompany them to the room using a close communicative attitude. Once in the room, the researcher woman stood next to the participant, where he or she had decided to sit, with the body slightly oriented towards him or her to favor the interaction and continue the conversation.

After the first informal conversation with the participants, they were shown the text they were going to read so that they could observe it and make their first reading hypotheses from the title. Later, they were explained that the objective of the activity was to read and understand the text and then be able to comment on the maximum possible aspects of their plot. For this, it was important that the participants were very focused on their reading and that they would go back if at any time they detected that they had got lost. Once the participants understood the instruction, they were encouraged to start reading aloud.

All data -informal conversation, instructions, reading aloud, commenting on different aspects of the plot with the researcher- were recorded with an audio recorder. In addition, a sketch of the body behaviors (proxemics of the participants with the text: distance of the head, trunk, arms and hands and angle) was made in a field notebook and it was noted at what time the text was produced, in each case. Then, an emptying of the proxemic categories that were detected in the sketches of the researcher was made and, in the end, the frequency of occurrence of each of the proxemic combinations in each course, school and reading proficiency level was studied.

\section{Results}

Figure 1 shows an approximate drawing of the four proxemic categories that could be identified and labeled in the sketches of the field notes; namely: 1) moving away, 2) leaning out, 3) approaching the text and 4) appropriating the text. Each of these categories appears with two or three combinations or varieties; from now on: strategies -like: diagonally, backwards, without supports, etc.- that give information about the position of hands, arms and trunk in the act of reading. In our corpus of sketches, up to ten different proxemic strategies were detected:

- Moving away diagonally from text

- Moving back from text

- Leaning out the text by booking at it without support

- Leaning out the text by looking at it and setting the hands and/or elbows on the table [+/- finger-pacer]

- Approaching the text surrounding it

- Approaching the text by holding it or touching it in the margins [+/- finger-pacer]

- Approaching the text by covering it [+/- finger-pacer]

- Appropriating the text by picking it slightly up

- Appropriating the text by raising it [+/- finger-pacer] 
- Appropriating the text by bringing it closer

As can be seen, the different strategies provide information on various dimensions of proxemic notation, while contemplating whether the interaction with the text is done face-to-face or sideways (such as moving away diagonally or moving backwards), defining the distance of the head, the trunk, the elbows and the hands and how they approach or interact (leaning on the table, surrounding the text, holding the text, covering the text, picking it slightly up, raising it or bringing it closer to the eyes) and allow description of the field of vision that is obtained from each behavior.

It should also be noted that some strategies, such as: looking at the text while setting the hands and/or elbows on the table; approaching the text by holding it with the fingers; approaching the text by covering it with the hands and/or arms; or appropriating the text by raising it upright, may or may not be accompanied by a finger-pacer. The action of following the lines of the text with the index finger or marking the beginning and end of each line with one finger of each hand is called the finger-pacer strategy (cf. Konstant 2003) $)^{15}$ to facilitate retinal jumps.

Without support

15 This strategy, according to Konstant (2003), helps increase reading speed and, as regards our study, increases the degree of activity in relation to the text. 


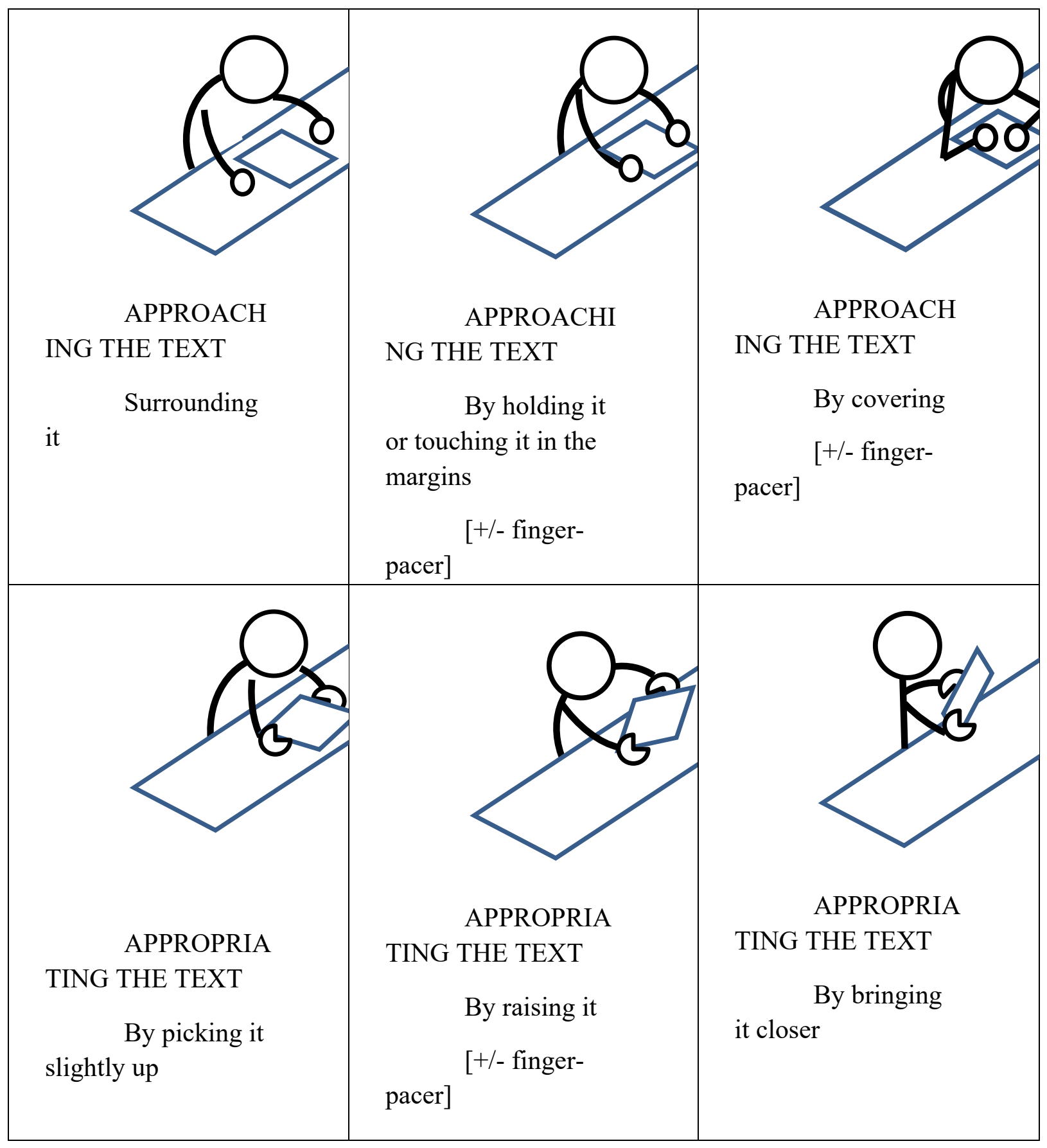

Figure 1. Images of proxemic strategies in primary readers

In the pages that follow, it is shown how often each of the proxemic categories are registered in the different strata of study of the sample: course, level of reading competence of the participants and school of origin. Before this, however, a description of the behavior of the data is offered generically.

Throughout the 72 reading tests, 86 posture changes were detected, since only $17 \%$ of the participants modified their proxemic behavior during the course of the reading activity. In this regard, the notes in the field diary explain that the readers who made the most proxemic changes used coincide with those that were least related to the text ...) in the pre-reading phase (observe it, touch it, get an idea of the extension, of the title, of the subject ...).

Only $5 \%$ of the participants adopted the proxemic category of maximum 
sociofugal (moving away). This is: contrary to favoring interaction with the text and to participating actively in reading. In the sections that follow, it will be verified that its appearance refers only to readers with a low level of competence. Also, it occurs only in one of the centers (center G).

In general, the category most used by primary school students is leaning out or looking at the text with and without support (41\%). This category is characterized by offering a wide field of vision and a very weak focusing capacity. In fact, it is easy to associate it with the reading of illustrated stories, catalogs or magazines, in which the image plays a relevant text. As will be seen in the following lines, this is the category that defines readers with a medium or low reading proficiency. And it will be decisive in the diagnosis of poor readers of the Middle and Higher Cycles. This category is rarely accompanied by a finger-pacer strategy (2.32\%). Finally, it should be said that $28 \%$ of the participants who used it made some change in strategy throughout their reading. These changes, for the most part, occurred from proxemic categories, a priori, more related to success and from the first third of the fragment read. This fact could be an indication that the leaning out category appears in situations of fatigue or loss in the process of understanding.

On the other hand, the categories of approaching the text and appropriating the text appear in a similar frequency order; $29 \%$ and $25 \%$ respectively and stand out especially in readers with a medium-high reading competence. Therefore, these categories will be substantially diagnostic of the good Initial Cycle readers. These are the categories that have the greatest opportunity to focus on the fragments read, and also, are the ones that best allow us to apply a finger-pacer strategy (follow the text with your fingers or indicate the jumps at the beginning and end of the line); especially in the case of approach. In $58 \%$ of cases in which the sample approaches the text is done by holding it or touching it in the margins. And in $64 \%$ of cases in which the sample appropriates the text, it does so by raising it. The most significant differences between one option and another are in the field of vision that is obtained and in the possibilities of applying a finger-pacer that focuses on the lines. The finger-pacer strategy, in fact, hardly appears associated with the appropriating the text category $(2.32 \%)$, but does, instead, with the category of approaching the text (especially by holding or touching the text in the margins). In general, however, the finger-pacer strategy is a resource rarely used by the sample and stands out above all in the readers with a medium-high reading competence of the Initial Cycle and in the readers with a medium-low competition of the Middle Cycle and Higher Cycle.

Graph 1 shows the percentages of occurrence of each proxemic category in each of the courses analyzed. It reflects that the category that appears most among the readers of the Initial Cycle is approaching the text, which appears in $36 \%$ of the cases registered in this course, followed by appropriating the text, which appears with a frequency of $35 \%$. In the Middle Cycle, the category that stands out the most is the leaning out one, which appears with a statistically significant difference: in $52 \%$ of cases. Finally, in the Higher Cycle, the categories that stand out the most are leaning out, which appears in $46 \%$ of the cases registered in this course, and approaching the text, which appears in $36 \%$. 


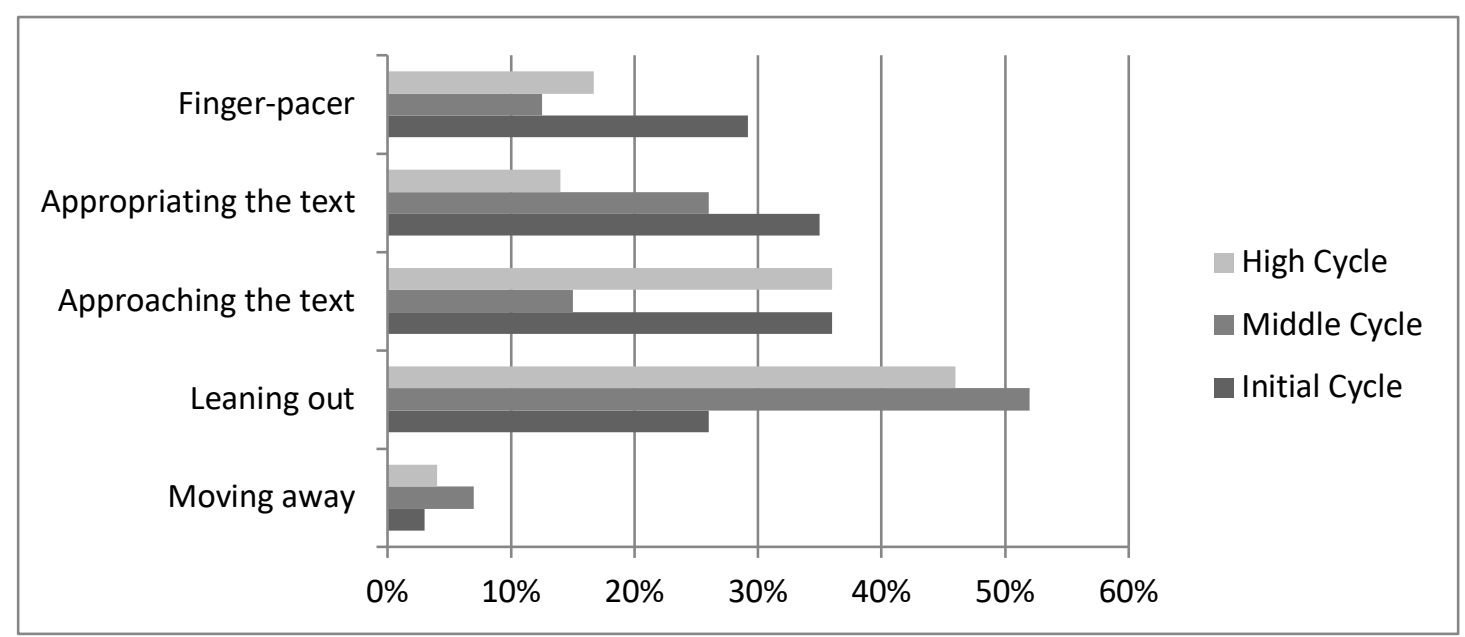

Graph 1. Relationship between proxemic categories and school year

The data in Graph 1 show that the students in the second year of primary school (Initial Cycle) maintain the proxemic behaviors that most favor contact with the text: approaching the text by holding it and appropriate the text by picking it slightly up or raising it. This type of strategy is accompanied in many cases by the use of the index finger as a pacer. It seems that in this Cycle the tendency is to actively interact with the text. It must be noted that, at this stage, reading activities receive more attention than other language activities, together with the transmission of reading habits, the relationship of schoolchildren with texts and literary enjoyment, and a constant reinforcement of decoding and reading automation skills. Hence, it coincides that the most used strategies in these courses are the ones that offer the most possibilities to focus on a fragment of the text, and more contact with the text facilitates.

The approach to the text is reduced by $21 \%$ of occasions in the readers of Middle Cycle and becomes a tendency to appropriate the text, although less than in the first case. However, the sample of Middle Cycle participants is the one that less actively relates to the text. This group stands out in the leaning out category; a category that also stands out among the readers with a medium-low level of competence of the Superior Cycle. This data could indicate the possibility that leaning out is a category that is in the zone of near development of the readers that begin to combine the abilities of focusing with the abilities of perception of the text as a unit. When this strategy is maintained, it is insufficient for the Higher Cycle, where successful readers come back to the text by holding it or either they appropriate the text by raising it.

By way of conclusion, the Initial Cycle readers are the ones that are most actively related to the text: they use the most sociopetal categories, with more kinaeshtetic and tactical strength and with more possibilities of focusing on read fragments (approaching the text and appropriating the text), while the Middle Cycle and Higher Cycle readers are those who relate more passively: they are situated in categories with little kinaesthetic and tactical strength and that hinder opportunities to focus the view on fragments of text (zoom out, lean out and approach the text surrounding it). In return, passive categories offer more field of vision.

Next, Graph 2 explores the different proxemics of readers according to the level 
of reading competence attributed to them by their teachers. The data does not allow us to describe a tendency in the frequency of appearance of the proxemic categories in the readers that, according to their teachers, have a high level of reading competence (A), although it can be affirmed that it is highly unlikely that a reader with this degree of reading competence would choose to move away from the text. The tendencies of appearance of some and other proxemic categories are identical in the group of readers with a medium competence (B) and in the group of readers with a low reading competence (C). These two levels of competence stand out in the leaning out category and show less predisposition than readers with a high reading proficiency level to appropriate the text. At this point, it may be convenient to study the emergence of different strategies (and not categories) at different levels of competence.

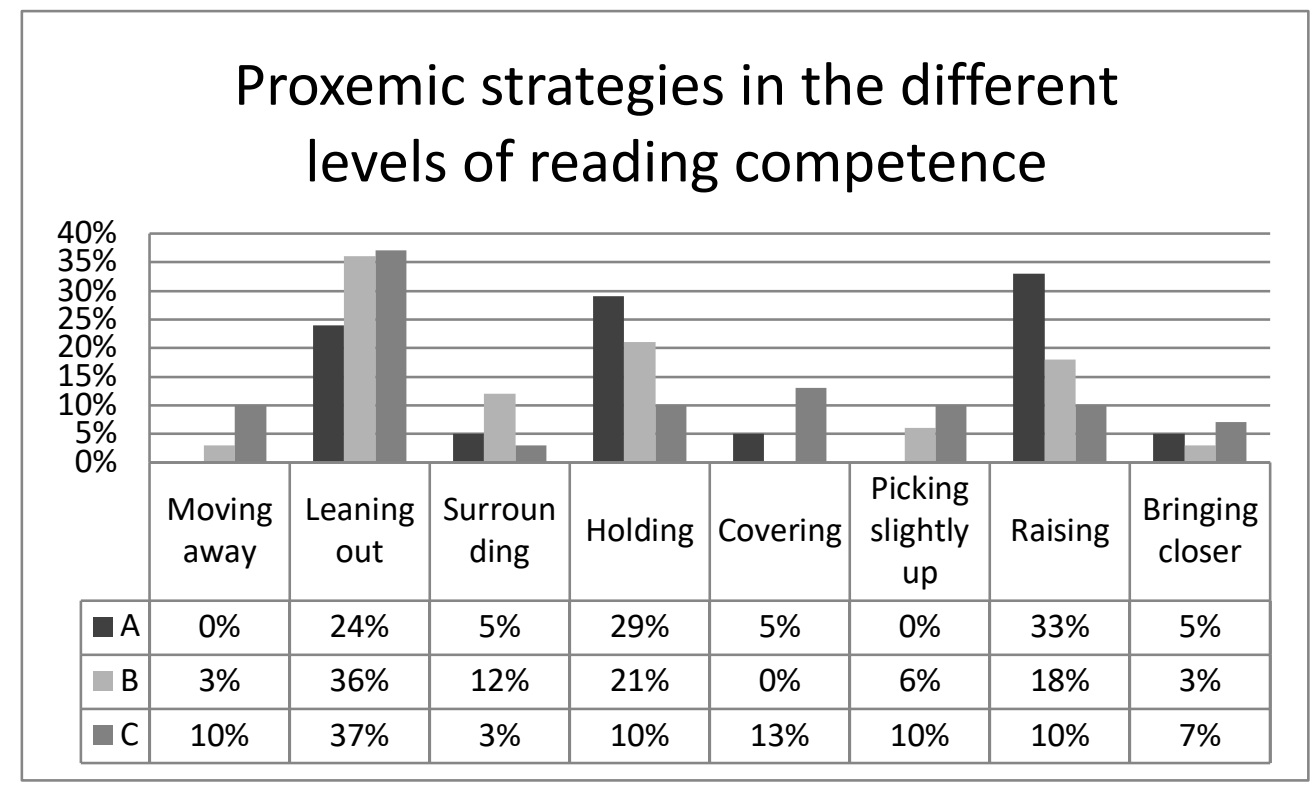

Graph 2. Proxemic strategies in the different levels of reading competence

Graph 2 throws new observations; for example, no reader with a high reading proficiency approaches the text by picking it slightly up, and very few choose to approach it by surrounding it or covering it. In fact, approaching the text by covering it seems to be an exclusive category of poor readers. With the exception of the category of moving away from the text and the strategy of appropriating the text by bringing it closer, which are uncommon patterns, all the strategies that good readers discard (surround the text, cover it, raising it) have a common characteristic: they offer a weak field of vision. In this regard, it should be noted that, according to Campayo (2009):

To read correctly you have to open the peripheral field of vision and avoid at all costs looking exclusively at what we have in front of our nose. In other words, we must open our lateral field of vision trying to emulate a kind of fisheye (p.73).

And, in effect, the strategy preferred by good readers -approaching the text by raising it-allows them to open the field of vision. The doubts appear with the approach of holding the text, which a priori does not seem to have this quality and yet it appears in a noticeable way in readers with a high reading competence and in readers with a medium reading competence; the category of leaning out, despite offering a broad field of vision, appears relevant to readers with medium reading competence and low reading proficiency. 
If readers with high proficiency stand out with strategies such as holding or raising the text, readers with low reading proficiency stand out in strategies such as looking at the text or covering it (which, in a way, is like looking very close, with greater focus and no field of vision). Something is clear: in the most used strategies, the levels of reading competence are distributed in an order of appropriation: less appropriation of the text is more for users with a low reading competence; more appropriation of the text is more for users with a high reading competence.

To conclude this section, the frequency of occurrence of each category in each of the schools in the sample is studied. In Graph 3, it is observed that in center L there is no proxemic strategy of the category moving away diagonally and that this category is not frequent either in center $\mathrm{G}$. With regard to the strategies of the category leaning out, in school $\mathrm{G}$ both strategies -setting the hands and/or the elbows on the table and without support-are common; while in school L it is much more frequent for readers to look at the text without support. The strategies of the category approaching the text appear in both centers, although it stands out in center $G$, along with the strategy of holding or covering it, compared to the strategy of surrounding it, which stands out in center L. Finally, in the category of appropriating the text, strategies of picking it slightly up, raising it and bringing it closer appear in a decreasing way, with the notable exception of the strategy of raising it, which is very frequent in the school G. Therefore, it seems that the context-school influences the predisposition to use particular strategies.

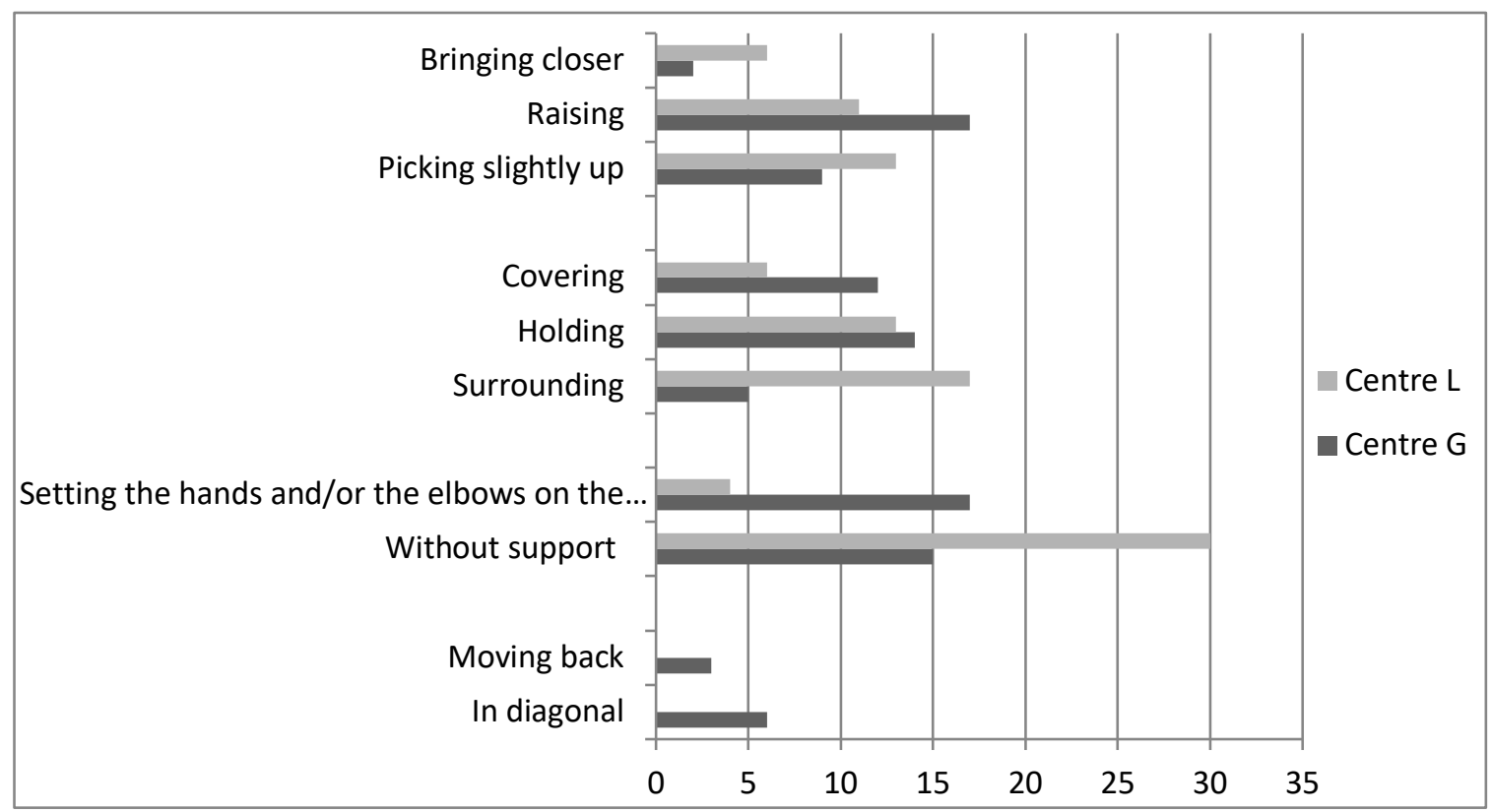

Graph 3. Relationship between proxemic categories and school context

\section{Discussion and conclusions}

Category 1, moving away, suggests, on the one hand, aversion to the text because it has very little force of appropriation, socio-petal orientation or kinaesthetic and touch activity. On the other hand, this category suggests possible sight problems, since when the reader moves away from the text, ends up getting less foveal contact with it. The fact of moving away from the text entails null possibilities of having contact with it, an also, 
to focus on the fragments read. This category could be compared to an attitude of backing down in an interaction between people, although, as we have seen, it could also indicate vision problems.

The field of vision is also wide in category 2, leaning out. That allows little focus on the retina, but, in return, this category increases the degree of proximity relationship to the text. The category 3, approaching the text, scores strongly in all dimensions, except in the field of vision. Finally, the readers who 4) appropriate the text (picking it slightly up, lifting it or bringing it closer) are taking possession of the object with which they interact, and that would be an example of greater appropriation and ability to focus on the retina.

The different strategies appear, in fact, ordered according to the appropriation that the readers maintain with the text -from no appropriation at all, in moving away diagonally, to a maximum appropriation, in appropriating the text by bringing it closerA priori, it could be understood that the degree of appropriation of the text is the result of combining, in a balanced way, a good level of sociopetality with the text, a good level of kinaesthetic and touch activity, an amplitude in the field of vision and a high possibility of focusing on the lines of the text that are being read. However, it is convenient to analyze the specific advantages of each strategy to go deeper into detail.

If the strategies are ordered according to the degree of closeness that the shoulders express with the text, the most socio-fugitive strategies could be considered as: moving away diagonally, moving backwards and appropriating the text by picking it slightly up. However, the most sociopetal ones would be appropriating the text by bringing it closer, appropriating the text by raising it and approaching the text by covering it. It is noteworthy that appropriating the text by picking it slightly up is a sociofugal strategy (which implies a greater angle of distance from the shoulders and, therefore, favors little interaction with the text) with respect to other forms of appropriation or with respect to the strategy of approaching the text covering it. From the point of view of the proximity of the shoulders with the object-text, therefore, the strategy of appropriating the text by picking it slightly up resembles the strategy of leaning out by looking at the text and setting the hands or elbows on the table.

And very much in relation to the distance at which the shoulders of the text object are placed, is the kinaesthetic factor (or degree of physical contact that is established with the text). The strategy of approaching the text by covering it, which was the third in the order of sociopetality, is the strategy with the greatest kinaesthetic factor, together with appropriating the text by bringing it closer and appropriating the text by raising it. Appropriating the text by picking it slightly up would be in the fourth place of kinaesthetic force, while the strategies with the lowest kinaesthetic factor would be the ones in the moving away and leaning out categories.

This order changes when what is contemplated is the force with which contact is established, although some consistency with the above is maintained. The strategies with a greater touch code are: appropriating the text by bringing it closer, appropriating the text by raising it and appropriating the text by picking it slightly up, followed by approaching the text by holding it or touching it in the margins and approaching the text by covering it. The strategies with the lowest touch codes are, again, the ones related to 
the categories of moving away and leaning out.

Finally, it is convenient to analyze these strategies from the retinal combinations they allow. If you look at the field of vision that can be obtained from each strategy, the categories that stand out the most are moving away and leaning out, and those that offer the least field of vision are those of the approaching the text category. Strategies in the appropriating the text category, then, are characterized by offering a medium field of vision. By contrast, if the strategies are analyzed from the possibility of focusing on certain parts of the text, the most suitable strategies are those of appropriating the text and approaching the text, and those that least help the reader in this aspect are moving away and leaning out.

In the following lines, a summary of the conclusions of this work is presented. Regarding the first objective of the work, to identify the proxemic categories and strategies of primary school readers, it is concluded that:

Readers have multiple proxemic strategies in reading. That is: not only do they follow the lines with their fingers or move their eyes and mouth, but they also relate to the body: moving away from the text, leaning out the text, approaching the text or appropriating the text with different strategies: diagonally, backwards, without supports, setting the hands and/or elbows on the table, surrounding the text, holding it or touching it in the margins, covering it, picking it slightly up, raising it or bringing it closer.

To describe the proxemics in the reading, variables such as the orientation of the shoulders, trunk, head and arms of the reader with respect to the text can be used; the quantity and quality of physical contact that occurs with the text and the angle of vision that is deduced from combining the other variables. These dimensions were described by Hall (1963) to observe the proxemic behavior of two people interacting. It would be possible to delve into new dimensions that would better describe the interaction with objects and, in particular, texts.

Regarding the second objective, to analyze the frequency in which each of the proxemic categories appears in the sample, we conclude that:

Most primary school readers lean out the text. This proxemics offers a very wide field of vision and a very weak focusing capacity. In the corpus, this proxemics appears associated with readers with a medium-low reading competence and situations of tiredness or loss in the comprehension process. It is very rare to find them in the moving away proxemics. On the other hand, the categories of approaching the text or appropriating the text appear in 25-30\% of cases. $16 \%$ of the cases described use the finger-pacer strategy at some time (or throughout the reading activity). This strategy usually appears in the readers who hold the text, and above all, among the students of the Initial Cycle. It would be useful to assess whether or not the students who use it obtain better comprehension results. The bibliography indicates that this strategy can help develop greater fluency.

Finally, when relating the proxemic categories with the course, reading proficiency level and school tradition, it is observed that:

As for the proxemics detected in the different courses, the participants who make 
the most changes in posture during the tests are part of the Initial Cycle and coincide with the participants who least related to the text (look at it, observe it, touch it) before starting to read. It is also concluded that in the Initial Cycle, the categories of approaching the text and appropriating the text, especially by subjecting it and applying a finger-pacer strategy or raising it stand out; that is, the categories that are closest to the text (sociopetal force, kinaesthetic force, touch force and focus of vision force). In the Middle Cycle, the strategy that stands out is looking at the text and appropriating the text by raising it; the use of the finger-pacer strategy significantly decreases here. Finally, in the Higher Cycle, the strategies of leaning out the text and approaching the text by holding it are more common, which makes it possible to use finger-pacers again.

If the different proxemics behaviors are related to the level of reading competence of schoolchildren, it is observed that the categories that are most described in the Initial Cycle coincide with the categories that stand out most in readers with a high reading proficiency. However, the categories that appear most in the Middle and Higher Cycle stand out among the readers with a medium-low level of competence. This is due, above all, to the weight that the leaning out strategy has in the last two Cycles.

No reader with high reading competence chooses to move away from the text or to appropriate it by picking it slightly up. In addition, it is infrequent to approach the text by surrounding it, cover it or appropriate the text by bringing it closer. This type of reader prefers to appropriate the text by raising it or approach it to hold it. Raising the text is one of the proxemic strategies that has the most appropriation, sociopetal, kinaesthetic, touch and focus force. And unlike approaching and holding the text, raising the text allows for a greater field of vision. On the other hand, the strategy of approaching the text and holding it punctuates with average values in all the proxemic dimensions of study, so it seems to be very useful and balanced for the reader. At the other extreme, readers with a low reading proficiency stand out in the leaning out category, although, as we have seen, this is a category that abounds at all levels. Also, the readers with a low reading competence usually approach the text by covering it. Finally, it is in this group that cases of moving away from the text are recorded.

In summary, the most successful strategies, or the ones that best describe readers with a high reading proficiency, are to approach the text by holding it and appropriating the text by raising it. By contrast, moving away from the text, appropriating the text by picking it slightly up and approaching the text by covering it are ineffective strategies. The most common category, leaning out, appears in different types of readers of various courses, but it stands out above all in the readers with a medium-low reading competence. It could be said that the more appropriative the proxemic category is, the more common it is to find it among readers of high reading competence and vice versa: the less appropriative the category is, the more common it is to find it among readers of low reading competence. In fact, readers with a low reading proficiency in the Higher Cycle do not appropriate the text. Finally, it should be noted that the strategies of approaching the text by surrounding it and appropriating the text by bringing it closer do not distinguish readers with greater or lesser level of reading proficiency, but readers of one and another school year: Higher and Initial respectively.

As regards the relationship between proxemic behaviors and school culture, there 
are evidences that the affective context that involves the reading activity, as well as the proxemics behavior of people who act as reading models for children, could influence the proxemic behavior of the learner. In fact, it is known that an important part of the initial learning of reading constitutes a game of imitation of the act of reading (based on models). In this initial game, very young readers assume that reading includes picking up some written material and looking at it; this is: the first thing that is fixed is in the body behavior and the person-text interaction. Perhaps, this game also continues in primary school, where the school is gradually related to new genres, formats, strategies and reading voices. In center $\mathrm{G}$, it is common to find readers who look at the text setting their hands or elbows on the table and readers who appropriate the text by raising it. Only in this center did we find cases of students moving away from the text. In the L center, readers who stand out without support and who approach the text around it stand out more.

\section{References}

Aguado, J. C. (2017). Análisis etnológico de la proxemia de un grupo otomí migrante a la Ciudad de México. Alteridades, 27(53), 83-94. Recovered August 20th, 2018 from: $\quad$ http://www.scielo.org.mx/scielo.php?script=sci arttext\&pid=S018870172017000100083\&lng=es\&nrm=iso

Birdwhistell, R. L. (1952). Introduction to kinesics. Louisville: University of Louisville. Campayo, R. (2009). Curso definitivo de lectura rápida: Método Ramón Campayo. Madrid: Edaf.

Cardellino, P., \& Araneda, C. (2014). Mapeo Vivencial del Fenómeno EnseñanzaAprendizaje en el Espacio de Aula Escolar-El Caso de Uruguay. Blucher Design Proceedings, 1(8), 144-147.

Castro, M. D. M. (2013). La cortesía: códigos verbales y no verbales en la comunicación interpersonal/Courtesy: verbal and non-verbal codes at interpersonal communication. Historia Y Comunicación Social, 18, 365-375. Recovered August 20th, 2018 from: http://revistas.ucm.es/index.php/HICS/article/view/44248/41811

Centro Virtual Cervantes (1997). "Proxémica" [online]. Diccionario De Términos clave de ELE. Madrid: Instituto Cervantes. Recovered January 8th, 2018 from: http://cvc.cervantes.es/ensenanza/biblioteca_ele/diccio_ele/diccionario/proxemi ca.htm

Crowder, R., \& Wagner, R. (1992). The psychology of reading: An introduction (2nd ed.). New York, US: Oxford University Press.

Erickson, F. (1975). One function of proxemic shifts in face-to-face interaction. En Kendon A., Harris, R.H., \& Ritchie Key, M. (Eds.). Organization of behaviour in face-to-face interactions. (pp. 115-187). Chicago: Aldine.

Guamán, A. D. (2016). Recuperación de hábitos y tradiciones de vida a través de la organización del espacio urbano en las ciudades. (Trabajo de graduación para optar al Título de Arquitecto). UCE, Quito. Recovered August 20th, 2018 from: http://www.dspace.uce.edu.ec/handle/25000/9514

Hall, E. T. (1963). A system for the notation of proxemic behavior. American Anthropologist, 65(5), 1003-1026. Recovered January 11th, 2018 from: 
http://onlinelibrary.wiley.com/doi/10.1525/aa.1963.65.5.02a00020/abstract

Hall, E. T. (1968). Proxemics. Current Anthropology, 9(2/3), 83-108. Recovered January 11th, 2018 from: http://www.journals.uchicago.edu/doi/abs/10.1086/200975

Huey, E. B. (1908). The psychology and pedagogy of reading. Cambridge, MA: M.I.T Press.

Knapp, M. (1982). La comunicación no verbal: El cuerpo y el entorno. Barcelona: Paidós.

Konstant, T. (2003). Técnicas de lectura rápida para leer más rápido y sacar más provecho de la lectura. Barcelona: Amat editorial.

Kusajima, T. (1974). Visual reading and braille reading: An experimental investigation of the physiology and psychology of visual and tactual reading. New York, US: American foundation for the Blind.

Mcconkie, G. (1976). The use of eye-movement data in determining perceptual span in reading. In Monty, R. A., \& Senders, J. W. (Eds.). Eye movements and psychological processes. Hillsdale, NJ: Lawrence Erlbaum.

Messi, V. (2015). Proxemia: análisis sociotécnico de una obra de arte electrónico. In: Alsina, P., \& Rodríguez, A. (Coord). «Art Matters II». Artnodes, 16, 34-42. UOC. Recovered August 20th, 2018 from: http://journals.uoc.edu/ojs/index.php/artnodes/article/view/n16-messi/n16messi-pdf-es

Poyatos, F. (1994). La comunicación no verbal II. Paralenguaje, kinésica e interacción. Madrid: Istmo.

Rayner, K., \& Sereno, S. (1994). Regressive eye movements and sentence parsing: On the use of regression-contingent analyses. Memory \& Cognition, 22, 281-285. Recovered January 10th, 2018 from: https://link.springer.com/article/10.3758/BF03200855

Recio-Pineda, S. (2016). Los lectores también tienen cuerpo. En A.E. Díez Mediavilla, V. Brotons Rico, D. Escandell, J. Rovira-Collado (coord.), Aprendizajes plurilingües y literarios: Nuevos enfoques didácticos (p. 751-760).

Rosa, A., \& Huertas, J. A. (1998). Peculiaridades de la lectura táctil del braille: Un estudio empírico. Infancia y Aprendizaje, 41, 79-94. https://doi.org/10.1080/02103702.1988.10822191

Sommer, R. (1969). Personal space: The Behavioral Basis of Design. Englewood Cliffs, N.J: Prentice-Hall (Spectrum).

Watson, O. M. (1972). Proxemics as non-verbal communication. In Ghosh, S. K. (Ed.). Man, language and society: Contributions to the sociology of language (pp 224231). The Hague: Mouton. 